\title{
WiLE: a Mathematica package for weak coupling expansion of Wilson loops in ABJ(M) theory
}

\author{
M. Preti ${ }^{1}$ \\ DESY Hamburg, Theory Group, Notkestraße 85, 22607 Hamburg, Germany
}

\begin{abstract}
We present WiLE, a Mathematica ${ }^{\circledR}$ package designed to perform the weak coupling expansion of any Wilson loop in $\mathrm{ABJ}(\mathrm{M})$ theory at arbitrary perturbative order. For a given set of fields on the loop and internal vertices, the package displays all the possible Feynman diagrams and their integral representations. The user can also choose to exclude non planar diagrams, tadpoles and self-energies. Through the use of interactive input windows, the package should be easily accessible to users with little or no previous experience. The package manual provides some pedagogical examples and the computation of all ladder diagrams at three-loop relevant for the cusp anomalous dimension in $\mathrm{ABJ}(\mathrm{M})$. The latter application gives also support to some recent results computed in different contexts.
\end{abstract}

\footnotetext{
${ }^{1}$ E-mail: michelangelo.preti@desy.de
} 


\section{Program Summary}

Author: Michelangelo Preti

Title: WiLE

Licence: GNU LGPL

Programming language / external routines: Wolfram Mathematica version 8.0 or higher Operating system: cross-platform

RAM: > 1 GB RAM recommended

Current version: 1.0 .0

Nature of the problem: In $\operatorname{ABJ}(\mathrm{M})$ theory one can define bosonic and fermionic Wilson loops. In order to study those operators using the standard perturbation theory techniques, one has to expand the Wilson loops at weak coupling and perform all the Wick contractions. Since the theory possesses a quiver structure and its Lagrangian contains many different vertices, this procedure could be very involved. Then we propose a Mathematica package to automatize this computation.

Solution method: The aim of the package WiLE is to obtain the weak coupling expansion of the Wilson loops in terms of Feynman diagrams. Given a set of initial data, as the set of vertices involved in the diagram and the fields contributing in the Wilson loop expansion, the package computes the related diagrams and their integral representations.

Web page: https://github.com/miciosca/WiLE

Contact: michelangelo.preti@desy.de - for bugs, possible improvements and questions

\section{Introduction}

Wilson loops are one of the most general gauge invariant observables in gauge theories and they play an important role in studying their general structure, both at perturbative and nonperturbative level. Depending on the contour, they encode different physical quantities, such as the potential between heavy moving probes or some aspects of scattering amplitudes of charged particles $[1,2,3]$. Furthermore, they are the building blocks of lattice formulation of any gauge theory, where they can be also used to study non-perturbative phenomenons. Remarkably, in the AdS/CFT framework [4, 5, 6], a new interest for Wilson loop in supersymmetric theories has been triggered. Indeed, they provide a rich class of BPS observables in supersymmetric gauge theories playing a central role in testing the correspondence itself, due to their relation with the fundamental string or brane configurations present in the dual theory [7, 8]. For example, whenever their vev is exactly computable (summing the perturbative series or through non perturbative techniques as supersymmetric localization or integrability), they provide nontrivial functions of the coupling which interpolate between weak and strong coupling regime. This allows for exact tests of the AdS/CFT correspondence [9, 10].

In $\mathcal{N}=4$ Super Yang-Mills theory (SYM), it was found a complete equivalence between scattering amplitudes in the planar limit and a particular class of null-polygonal Wilson loops in twistor space [11]. Other similar relation was proposed in [12, 13]. Furthermore, dualities between Wil- 
son loops and scattering amplitudes at weak and strong coupling have played a crucial role in understanding the integrable structure underlying both the gauge theory and its string dual (see $[14,15,16])$. Correlation functions of Wilson loops and local operators was also computed exactly using supersymmetric localization and perturbation theory [17, 18, 19] (see [20] for a review). Recently, also Wilson loops with operator insertions were successfully studied both at weak and strong coupling using integrability [21, 22] and localization techniques [23], enlightening their role in the context of defect CFTs [24, 25]. Similar properties have also emerged in other superconformal theories as $\mathcal{N}=6$ Super Chern-Simons-matter (or $\mathrm{ABJ}(\mathrm{M})$ ) theory in three dimensions [26, 27].

Bosonic Wilson loops in $\mathrm{ABJ}(\mathrm{M})$ theory with gauge group $U(N) \times U(M)$ can be defined [28] as the holonomy of a generalized gauge connection. This includes the coupling with scalars $C$ and $\bar{C}$ via a matrix locally defined along the path. When the path is a maximal circle of $S^{2}$ or an infinite line, the Wilson loop is a 1/6 BPS operator (see also [28, 29, 30]). Fermionic Wilson loops can be constructed turning on local couplings to the fermions $\psi$ and $\bar{\psi}$, generalizing the operator to the holonomy of a superconnection of the $U(N \mid M)$ supergroup [31]. The supersymmetry of the latter is enhanced to $1 / 2$ BPS on a maximal circle or an infinite line contours (see also [32, 33]). The fermionic loop operator is cohomologically equivalent to a linear combination of bosonic Wilson loops [31]. Perturbative computations for bosonic and fermionic Wilson loops have provided several results as the circular Wilson loop [34, 35, 36], the cusp anomalous dimension $[37,38,39]$ and the Bremsstrahlung function [40, 41, 42]. These calculations are quite involved then, in order to simplify and automatize the weak coupling expansion in terms of Feynman diagrams, we have developed the Mathematica ${ }^{\circledR}$ package WiLE.

The purpose of the package WiLE is to obtain the weak coupling expansion of the Wilson loop in terms of Feynman diagrams and their related integral representations. The package includes three functions: WiLE, WiLEFullorder and WiLESimplify. The first function, for a given set of input data as the number and the type of interaction vertices and fields on the loop ${ }^{2}$, draws the related Feynman diagrams and shows the integral representations of any diagram in terms of propagators, position vectors and local couplings of the fields. The WiLEFullorder function gives the same output of the first one but it groups the complete expansion for a fixed perturbative order. Many other options can be chosen: the relevant diagrams in the large $N$ and $M$ limit can be selected, or particular classes as tadpoles or self-energies can be excluded. Finally, the WiLESimplify function provides the simplification of the output if needed.

The paper is organized as follows. In section 2 we review the bosonic and fermionic Wilson loops and their perturbative expansions. In section 3 we present in detail the manual of the WiLE package, while in section 4 we show an explicit applications. Few appendices follow, which contain conventions, some details of $\mathrm{ABJ}(\mathrm{M})$ action and Feynman rules and a detailed legend to interpret the package output.

\footnotetext{
${ }^{2}$ Expanding the Wilson loop operator up to a perturbative order $\ell$, we will end up with the product of $\ell$ (super-)connections. Each connection contributes as a gauge field, a fermion or a couple of scalars with their positions along the loop fixed by the path-ordering.
} 


\section{Wilson loops in $\mathrm{ABJ}(\mathrm{M})$ theory}

In the $A d S_{4} / C F T_{3}$ correspondence framework, the Wilson loops are dual to string surfaces [7, 8]. Since in the most symmetric case the string preserves half of the supercharges of the vacuum, the dual Wilson loop operator in the field theory must be $1 / 2$ BPS. In analogy with the $\mathcal{N}=4$ SYM theory, the most natural choice is to consider a bosonic Wilson loop as the one defines in the $\mathcal{N}=2,3$ superconformal Chern-Simons-matter theories [43]. This loop was studied in the context of $\mathrm{ABJ}(\mathrm{M})$ in $[28,29,30]$ but, since it preserves only $1 / 6$ of the supercharges (if it lie on a straight line or circular contour), it cannot be a viable candidate to be the dual of the classical string. In [31], the authors proposed a new Wilson loop operator that couples both to the bosonic and fermionic fields of the theory using explicitly the quiver structure of the theory. This operator turns out to be $1 / 2$ BPS (if it lies on a straight line or circular contour) and it is dual to the $1 / 2$ BPS string solution found in $[28,30]$. There are more general BPS Wilson loops, lying along arbitrary curves and preserving fewer supersymmetries. These loops have been proposed in [44] and they generalize the straight line and the circle studied in [31]. Clearly it is possible to consider also non-supersymmetric operators as, for instance, Wilson loops with cusps or selft-intersections.

In this section we review the bosonic and fermionic Wilson loop operators in ABJ(M) theory and their weak coupling expansion.

\subsection{The bosonic Wilson loops}

In ABJM theory there are quite a few possibilities to construct gauge-invariant Wilson loop operators: one choice would be simply the standard Wilson loop operators which are defined respect to the gauge holonomies $W_{A}$ and $\hat{W}_{A}$ of the $U(N)_{\kappa}$ and $U(M)_{-\kappa}$ groups respectively as follows

$$
\begin{aligned}
& \mathcal{W}_{A}[C]=\frac{1}{N} \operatorname{Tr}_{\mathbf{N}}\left(W_{A}[C]\right)=\frac{1}{N} \operatorname{Tr}_{\mathbf{N}} \mathcal{P} \exp \left[-i \int_{C} d \tau A_{\mu} \dot{x}^{\mu}\right], \\
& \hat{\mathcal{W}}_{A}[C]=\frac{1}{M} \operatorname{Tr}_{\mathbf{M}}\left(W_{A}[C]\right)=\frac{1}{M} \operatorname{Tr}_{\mathbf{M}} \mathcal{P} \exp \left[-i \int_{C} d \tau \hat{A}_{\mu} \dot{x}^{\mu}\right],
\end{aligned}
$$

where $x^{\mu}(\tau)$ parametrizes the curve $C$ along which the loop operator is supported, $\operatorname{Tr}_{\mathbf{N}}$ and $\operatorname{Tr}_{\mathbf{M}}$ represent the color traces taken over the fundamental of $U(N)_{\kappa}$ and $U(M)_{-\kappa}$ respectively and $\mathcal{P}$ is the path-ordering operator.

The loop operators (1) are not BPS. In order to have an operator locally BPS at least, one has to define the connection with some coupling to the scalars. The resulting operators are

$$
\begin{aligned}
& \mathcal{W}_{B}[C]=\frac{1}{N} \operatorname{Tr}_{\mathbf{N}}\left(W_{B}[C]\right)=\frac{1}{N} \operatorname{Tr}_{\mathbf{N}} \mathcal{P} \exp \left[-i \int_{C} d \tau\left(A_{\mu} \dot{x}^{\mu}-\frac{2 \pi i}{\kappa}|\dot{x}| M_{J}{ }^{I} C_{I} \bar{C}^{J}\right)\right], \\
& \hat{\mathcal{W}}_{B}[C]=\frac{1}{M} \operatorname{Tr}_{\mathbf{M}}\left(\hat{W}_{B}[C]\right)=\frac{1}{M} \operatorname{Tr}_{\mathbf{M}} \mathcal{P} \exp \left[-i \int_{C} d \tau\left(\hat{A}_{\mu} \dot{x}^{\mu}-\frac{2 \pi i}{\kappa}|\dot{x}| \hat{M}_{J}{ }^{I} \bar{C}^{J} C_{I}\right)\right],
\end{aligned}
$$

where $M_{J}{ }^{I}$ and $\hat{M}_{J}{ }^{I}$ in general are matrices with arbitrary entries but that can be constrained 
by supersymmetry.

Using the supersymmetry transformation (95), one can consider the variation of the operators (2) under supersymmetry and impose that it vanishes for a suitable choice of the parameter $\theta$. For instance, considering the first operator of (2), one finds the following condition

$$
\begin{aligned}
\delta_{\theta} \mathcal{W}_{B} \sim \theta^{I J \alpha}\left[-\dot{x}_{\mu} \sigma_{\alpha \beta}^{\mu} \delta_{I}^{P}+|\dot{x}| \delta_{\alpha \beta} M_{I}{ }^{P}\right] C_{P}\left(\psi_{J}\right)^{\beta} & \\
& +\epsilon_{I J K L} \theta^{I J \alpha}\left[\dot{x}_{\mu} \sigma_{\alpha \beta}^{\mu} \delta_{P}^{K}+|\dot{x}| \delta_{\alpha \beta} M_{P}{ }^{K}\right]\left(\bar{\psi}^{L}\right)^{\beta} \bar{C}^{P}=0
\end{aligned}
$$

In order to have a supersymmetric operator, both terms in the formula above have to vanish separately. The choice of which supercharges are preserved by the operator completely fixes the scalar coupling $M_{J}{ }^{I}$ and the geometry of the contour $\dot{x}^{\mu}$ and vice-versa. Indeed if the contour is a straight line, the Wilson line operator is invariant under two of the 12 Poincaré supersymmetries and two of the 12 superconformal supersymmetries, i.e. the loop is $1 / 6 \mathrm{BPS}^{3}$.

In general, one can take any combination of $\mathcal{W}_{B}[C]$ and $\hat{\mathcal{W}}_{B}[C]$ in any representation of each of the gauge groups. An explicit computations in planar perturbation theory [28, 30] shows that the operator identifiable with the appropriate Type IIA fundamental string configurations ${ }^{4}$ is the sum of $\mathcal{W}_{B}$ and $\hat{\mathcal{W}}_{B}$.

\subsection{The fermionic Wilson loop}

The idea of [31] is to define the $1 / 2$ BPS Wilson line (or circular Wilson loop) ${ }^{5}$ embedding the gauge connections of $U(N)_{\kappa}$ and $U(M)_{-\kappa}$ into a superconnection of the form

$$
\mathcal{L}(\tau) \equiv\left(\begin{array}{cc}
A_{\mu} \dot{x}^{\mu}-\frac{2 \pi i}{k}|\dot{x}| \mathcal{M}_{J}{ }^{I} C_{I} \bar{C}^{J} & i \sqrt{\frac{2 \pi}{k}}|\dot{x}| \eta_{I} \bar{\psi}^{I} \\
-i \sqrt{\frac{2 \pi}{k}}|\dot{x}| \psi_{I} \bar{\eta}^{I} & \hat{A}_{\mu} \dot{x}^{\mu}-\frac{2 \pi i}{k}|\dot{x}| \hat{\mathcal{M}}_{J}{ }^{I} \bar{C}^{J} C_{I}
\end{array}\right)
$$

belonging to the super-algebra of $U(N \mid M)$. The matrices $\mathcal{M}_{J}^{I}$ and $\hat{\mathcal{M}}_{J}{ }^{I}$ and the Grassmann even quantities $\eta_{I}^{\alpha}$ and $\bar{\eta}_{\alpha}^{I}$ parameterize the possible local couplings. The above supermatrix $\mathcal{L}$ is in general $(N+M) \times(N+M)$ : the upper-left block is $N \times N$ and the lower-right is $M \times M$. In particular, the diagonal entries have the same structure of the gauge connections of the bosonic Wilson loops $\mathcal{W}_{B}[C]$ and $\hat{\mathcal{W}}_{B}[C]$ respectively discussed above with different scalar couplings. For a given path $C$, the holonomy of the superconnection (4) is defined as

$$
W[C] \equiv \mathcal{P} \exp \left(-i \int_{C} d \tau \mathcal{L}(\tau)\right)
$$

In the AdS/CFT framework it is useful to require that the Wilson loop possesses a local $U(1) \times$

\footnotetext{
${ }^{3}$ The line can be mapped to a circle under a conformal transformation. Then the circular Wilson loop possesses the same number of supersymmetries of the Wilson line but, since such transformation mixes the super-Poincaré and superconformal charges, it is invariant under a linear combination of them.

${ }^{4}$ The fundamental string ending along a straight line on the boundary of $A d S_{4}$ and localized on $\mathbb{C P}^{3}$ preserves 12 supercharges. In order to match with the gauge theory observable one has to smear the string over a $\mathbb{C P}^{1}$, breaking indeed the supersymmetry down to $1 / 6$.

${ }^{5}$ All the statements of this section are true for a generic contour but the operator will preserves less supercharges.
} 
$S U(3)$ R-symmetry invariance as the semi-classical string surface. This requirement restricts the coupling to be of the form

$$
\eta_{I}^{\alpha}=n_{I} \eta^{\alpha}, \quad \bar{\eta}_{\alpha}^{I}=\bar{n}^{I} \bar{\eta}_{\alpha}, \quad \mathcal{M}_{J}^{I}=p_{1} \delta_{J}^{I}-2 p_{2} n_{J} \bar{n}^{I}, \quad \hat{\mathcal{M}}_{J}^{I}=q_{1} \delta_{J}^{I}-2 q_{2} n_{J} \bar{n}^{I}
$$

where the reduced vector coupling $n_{I}$ (and its complex conjugate $\bar{n}^{I}$ ) define the embedding of the unbroken $S U(3)$ subgroup into $S U(4)$ and it can be chosen such that $n_{I} \bar{n}^{I}=1$. The free parameters $p_{i}(\tau)$ and $q_{i}(\tau)$, and the reduced spinor couplings can be constrained by requiring that the Wilson loop defined by the superconnection (4) is globally supersymmetric up to a super-gauge transformation ${ }^{6}$

$$
\delta_{\theta} \mathcal{L}(\tau)=\mathcal{D}_{\tau} G \equiv \partial_{\tau} G+i\{\mathcal{L}, G]
$$

with $G$ an anti-diagonal supermatrix. The resulting couplings are

$$
\begin{gathered}
\left(\dot{x}^{\mu} \gamma_{\mu}\right)_{\alpha}{ }^{\beta}=\frac{\ell}{2 i}|\dot{x}|\left(\eta^{\beta} \bar{\eta}_{\alpha}+\eta_{\alpha} \bar{\eta}^{\beta}\right), \quad\left(\eta^{\beta} \bar{\eta}_{\alpha}+\eta_{\alpha} \bar{\eta}^{\beta}\right)=2 i \delta_{\alpha}^{\beta}, \\
\mathcal{M}_{J}^{I}=\hat{\mathcal{M}}_{J}^{I}=\ell\left(\delta_{J}^{I}-2 n_{J} \bar{n}^{I}\right),
\end{gathered}
$$

where $\ell= \pm 1$. Notice that the vectors $n_{I}$ and $\bar{n}^{I}$ are still unconstrained.

In order to determine the number of supersymmetries preserved by a certain fermionic Wilson loop, one has to solve the general set of BPS conditions given in [44] using the following ansatz for the reduced vector couplings

$$
\bar{n}^{I}=r\left(\eta U \bar{s}^{I}\right) \quad \text { and } \quad n_{I}=\frac{1}{r}\left(s_{I} U^{-1} \bar{\eta}\right)
$$

where $s_{I}^{\alpha}$ and $\bar{s}_{\alpha}^{I}$ are $\tau$-independent spinors obeying the completeness relation $\bar{s}_{\beta}^{I} s_{I}^{\alpha}=\frac{1}{2 i} \delta_{\beta}^{\alpha}$, and

$$
r=r_{0} e^{-\frac{i \tau}{2} \sin 2 \alpha} \text { and } \quad U=\cos \alpha \mathbb{1}+i \sin \alpha\left(x^{\mu} \gamma_{\mu}\right),
$$

with $\alpha$ free constant parameter. The resulting couplings are the following

$$
\begin{aligned}
\eta_{I}^{\beta} & =\frac{i}{r_{0}} e^{\frac{i}{2}(\sin 2 \alpha) \tau}\left[s_{I}\left(\cos \alpha \mathbb{1}-i \sin \alpha\left(x^{\mu} \gamma_{\mu}\right)\right)\left(\mathbb{1}+\frac{\dot{x} \cdot \gamma}{|\dot{x}|}\right)\right]^{\beta}, \\
\bar{\eta}_{\beta}^{I} & =i r_{0} e^{-\frac{i}{2}(\sin 2 \alpha) \tau}\left[\left(\mathbb{1}+\frac{\dot{x} \cdot \gamma}{|\dot{x}|}\right)\left(\cos \alpha \mathbb{1}+i \sin \alpha\left(x^{\mu} \gamma_{\mu}\right)\right) \bar{s}^{I}\right]_{\beta}, \\
\mathcal{M}_{K}^{J} & =\hat{\mathcal{M}}_{K}^{J}=\left[\delta_{K}^{J}-2 i s_{K} \bar{s}^{J}-2 i \cos 2 \alpha\left(s_{K} \frac{\dot{x} \cdot \gamma}{|\dot{x}|} \bar{s}^{J}\right)-2 i \sin 2 \alpha\left(s_{K} \gamma^{\lambda} \bar{s}^{J}\right) \epsilon_{\lambda \mu \nu} x^{\mu} \dot{x}^{\nu}\right] .
\end{aligned}
$$

If the contour is parametrized in $\mathbb{R}^{3}$ the ansatz (9) has $\alpha=0$ and consequently the couplings (11). The associated Wilson loops are 1/12 BPS and they are the three-dimensional companions of the operators discussed in [45]. In this case the most supersymmetric operator is the $1 / 2 \mathrm{BPS}$ Wilson line. If the contour is parametrized on the sphere $S^{2}$, the parameter $\alpha$ is arbitrary and the associated Wilson loops are in general 1/12 BPS . It is interesting to mention that for $\alpha=0$ the scalars decouple from the 2 -forms on $S^{2}$ (i.e. the last term in the scalar couplings $\mathcal{M}$ and $\hat{\mathcal{M}}$ )

\footnotetext{
${ }^{6}$ The usual condition $\delta_{\theta} \mathcal{L}(\tau)=0$ gives rise only to bosonic Wilson loops $(\eta=\bar{\eta}=0)$ and at most $1 / 6$ BPS.
} 
and the corresponding Wilson loops can be interpreted as a deformation of the previous case. For $\alpha=\frac{\pi}{4}$, scalars couple only to the invariant forms and one can recover the $1 / 2$ BPS circle, the $1 / 6$ BPS latitude and the 1/6 BPS two-longitude. These Wilson loops can be interpreted as the three-dimensional companions of the operators discussed in $[46,47]$.

Finally one has to consider the boundary conditions obeyed by the gauge functions (i.e. the entries of the matrix $G$ defined in (7)) to obtain a gauge invariant object. If the holonomy is defined on a closed contour the Wilson loop operator is defined as the trace of (5). For an infinite open circuit, such as the straight line, there are two possible gauge-invariant operators

$$
\mathcal{W}_{-}[C]=\frac{1}{N-M} \mathrm{~S} \operatorname{Tr}[W[C]] \quad \text { and } \quad \mathcal{W}_{+}[C]=\frac{1}{N+M} \operatorname{Tr}[W[C]]
$$

where the trace and the supertrace are taken in the fundamental of $U(N)_{\kappa}$ and $U(M)_{-\kappa}$ for the $N \times N$ and $M \times M$ blocks of the supermatrix respectively.

\subsection{The Wilson loops weak coupling expansion}

The vacuum expectation value of a fermionic Wilson loop is given by the usual path integral representation

$$
\left\langle\mathcal{W}_{ \pm}[C]\right\rangle=\frac{1}{N \pm M} \int \mathcal{D}[\Psi] e^{-S_{\mathrm{ABJ}(\mathrm{M})}}(\mathrm{S}) \operatorname{Tr}[W[C]]
$$

where $\mathcal{D}[\Psi]$ specifies the integration over all the fields of the theory and $S_{\mathrm{ABJ}(\mathrm{M})}$ is the action of the $\operatorname{ABJ}(\mathrm{M})$ theory in the Euclidean space (see appendix B). The symbol (S)Tr stands for the trace or the supertrace respectively for $\mathcal{W}_{+}$and $\mathcal{W}_{-}$(see (12)).

Since the holonomy $W[C]$ is a supermatrix and one has to take its (super)trace, it is useful to split the vev (13) into the two diagonal partial vevs as follows

$$
\left\langle\mathcal{W}_{ \pm}[C]\right\rangle=\frac{\left\langle\mathcal{W}^{\uparrow}[C]\right\rangle \pm\left\langle\mathcal{W}^{\downarrow}[C]\right\rangle}{N \pm M}
$$

The partial vevs $\mathcal{W}^{\uparrow(\downarrow)}[C]$ are defined by

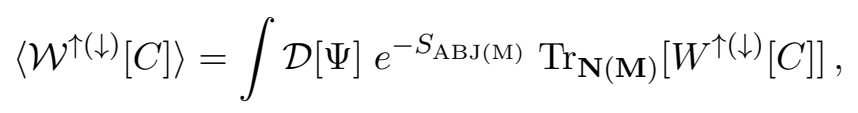

where the arrows $\uparrow$ and $\downarrow$ specify respectively the upper-left $N \times N$ and the lower-right $M \times$ $M$ blocks of the supermatrix $W[C]$. Recalling the definitions (4) and (5), the path-ordered 
expansion of $W^{\uparrow}[C]$ is given by ${ }^{7}$

$$
\begin{aligned}
\operatorname{Tr}_{\mathbf{N}}\left(W^{\uparrow}[C]\right)=\operatorname{Tr}_{\mathbf{N}}\left[\mathbb{1}_{N}-i \int_{C} d \tau_{1} \mathcal{A}_{1}-\int_{C} d \tau_{1>2}\left(\mathcal{A}_{1} \mathcal{A}_{2}+\left|\dot{x}_{1}\right|\left|\dot{x}_{2}\right|(\eta \bar{\psi})_{1}(\psi \bar{\eta})_{2}\right)\right. \\
+i \int_{C} d \tau_{1>2>3}\left(\mathcal{A}_{1} \mathcal{A}_{2} \mathcal{A}_{3}-\frac{2 \pi}{k}\left[\left|\dot{x}_{1}\right|\left|\dot{x}_{2}\right|(\eta \bar{\psi})_{1}(\psi \bar{\eta})_{2} \mathcal{A}_{3}\right.\right. \\
\left.\left.\left.\quad+\left|\dot{x}_{1}\right|\left|\dot{x}_{3}\right|(\eta \bar{\psi})_{1} \hat{\mathcal{A}}_{2}(\psi \bar{\eta})_{3}+\left|\dot{x}_{2}\right|\left|\dot{x}_{3}\right| \mathcal{A}_{1}(\eta \bar{\psi})_{2}(\psi \bar{\eta})_{3}\right]\right)+\ldots\right]
\end{aligned}
$$

where the subscripts specify the dependence of the fields from the position $x_{i}=x\left(\tau_{i}\right)$ and $\mathcal{A}$ and $\hat{\mathcal{A}}$ refers to the upper-left and lower-right diagonal blocks of the superconnection (4) resepctively. The spinor and R-symmetry indices are suppressed $\left(\eta \bar{\psi} \equiv \eta_{I}^{\alpha} \bar{\psi}_{\alpha}^{I}\right.$ and $\left.\psi \bar{\eta} \equiv \psi_{I}^{\alpha} \bar{\eta}_{\alpha}^{I}\right)$. The path-ordered integrals in (16) are defined as follows

$$
\int_{C} d \tau_{1>2>\ldots>n-1>n}=\int_{a}^{b} d \tau_{1} \int_{a}^{\tau_{1}} d \tau_{2} \ldots \int \ldots \int_{a}^{\tau_{n-2}} d \tau_{n-1} \int_{a}^{\tau_{n-1}} d \tau_{n}
$$

for $\tau_{i} \in[a, b]$. An interesting property of the partial vevs defined in (15) is

$$
\left\langle\mathcal{W}^{\uparrow}[C]\right\rangle \stackrel{N \leftrightarrow M}{=}\left\langle\mathcal{W}^{\downarrow}[C]\right\rangle
$$

that clearly drastically simplify the computation of (14).

The same considerations can be done for the bosonic Wilson loops. The diagonal entries of the superconnection (4) resemble the gauge connections of the bosonic Wilson loops $\mathcal{W}_{B}[C]$ and $\hat{\mathcal{W}}_{B}[C]$ defined in (2) but with different scalar couplings. Indeed the vev of the bosonic Wilson loops are different from the vev computed from the fermionic operator turning off the fermionic couplings (in some cases this equivalence is true, for example the circular Wilson loop at framing one $\left.{ }^{8}[31,51]\right)$. Since our purpose is to provide an algorithmic method to expand the Wilson loops at weak coupling, we don't need to specify the value of the scalar coupling at any time. The two bosonic Wilson loops are the trace of the holonomy of $U(N)$ and $U(M)$ and they are related to the upper-left and lower-right blocks of the superconnection (4) respectively. The expansion of bosonic loop operators can be reconstructed form the fermionic one (see (16)) with some simple substitutions. Indeed, setting the fermionic coupling $\eta$ and $\bar{\eta}$ to zero in the expansions of $\operatorname{Tr}_{\mathbf{N}}\left(W^{\uparrow}[C]\right)$ or $\operatorname{Tr}_{\mathbf{M}}\left(W^{\downarrow}[C]\right)$, and substituting the scalar couplings $\mathcal{M}_{J}^{I} \rightarrow M_{J}^{I}$, we obtain the expansion for the bosonic loops operator as follows

$$
\begin{aligned}
\operatorname{Tr}_{\mathbf{N}}\left(W_{B}[C]\right) & =\operatorname{Tr}_{\mathbf{N}}\left(W^{\uparrow}[C]\right)_{\eta=\bar{\eta}=0, \mathcal{M} \rightarrow M} \\
\operatorname{Tr}_{\mathbf{M}}\left(\hat{W}_{B}[C]\right) & =\operatorname{Tr}_{\mathbf{M}}\left(W^{\downarrow}[C]\right)_{\eta=\bar{\eta}=0, \mathcal{M} \rightarrow M}
\end{aligned}
$$

\footnotetext{
${ }^{7}$ The path ordered expansion of $W^{\downarrow}[C]$ is the same of $W^{\uparrow}[C]$ with the following replacements: $N \leftrightarrow M$, $\mathcal{A}_{i} \leftrightarrow \hat{\mathcal{A}}_{i}$ and $(\eta \bar{\psi})_{i} \leftrightarrow(\psi \bar{\eta})_{i}$

${ }^{8}$ In Chern-Simons theories the vev of Wilson loop operators on close paths could be affected by finite regularization ambiguities if it is used a point-splitting regularization for contact singularities which appear when multiple points collide. In this regularization scheme this can be avoided by requiring that every point $x_{i}$ runs on a different path called frame (for example in pure Chern-Simons theory see [48, 49, 50]).
} 
and for the operators $\mathcal{W}_{A}[C]$ and $\hat{\mathcal{W}}_{A}[C]$

$$
\begin{aligned}
\operatorname{Tr}_{\mathbf{N}}\left(W_{A}[C]\right) & =\operatorname{Tr}_{\mathbf{N}}\left(W^{\uparrow}[C]\right)_{\eta=\bar{\eta}=\mathcal{M}=\hat{\mathcal{M}}=0} \\
\operatorname{Tr}_{\mathbf{M}}\left(\hat{W}_{A}[C]\right) & =\operatorname{Tr}_{\mathbf{M}}\left(W^{\downarrow}[C]\right)_{\eta=\bar{\eta}=\mathcal{M}=\hat{\mathcal{M}}=0}
\end{aligned}
$$

Then one can read the weak coupling expansion of the bosonic Wilson loops from the fermionic one defined in (16).

In $\operatorname{ABJ}(\mathrm{M})$ theory the Chern-Simons level $\kappa$ plays the role of a coupling. Indeed one can define the coupling constant $g_{C S}^{2}=\frac{1}{\kappa}$ that plays a similar role of $g_{Y M}^{2}$ in $\mathcal{N}=4 \mathrm{SYM}$, albeit $\kappa$ has to be an integer to preserve non-abelian gauge symmetry. Then it is possible to study the vev of the Wilson loop with the usual perturbation theory technique at weak coupling $\left(g_{C S} \ll 1\right)$ as follows

$$
\left\langle\mathcal{W}^{\uparrow}[C]\right\rangle \equiv \sum_{\ell=0}^{\infty} g_{C S}^{2 \ell} a_{\ell}^{\uparrow}(N, M)=\sum_{\ell=0}^{\infty} \frac{a_{\ell}^{\uparrow}(N, M)}{\kappa^{\ell}}
$$

where using (18) we have $a_{\ell}^{\downarrow}(N, M)=a_{\ell}^{\uparrow}(M, N)$. The coefficients $a_{\ell}^{\uparrow}$ can be expressed in terms of Feynman diagrams. For $\ell=0,1$ we have

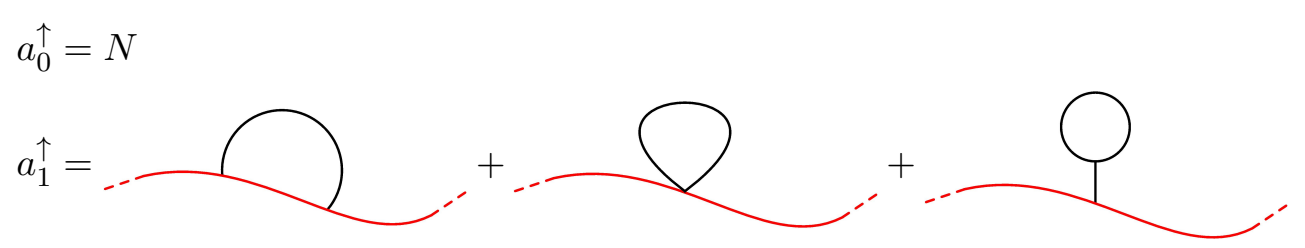

where the red line represent the Wilson loop and the black lines are propagators (it is not specified the type of the propagators because here we are interested only in the topology of the diagrams). Finally the observable can be restricted in the planar 't Hooft limit which introduces the following effective couplings

$$
\lambda_{1} \equiv g_{C S}^{2} N=\frac{N}{\kappa}, \quad \lambda_{2} \equiv g_{C S}^{2} M=\frac{M}{\kappa} \quad \text { with } \kappa, M, N \rightarrow \infty
$$

In the ABJM case, since $M=N$, the theory has only one coupling $\lambda=\lambda_{1}=\lambda_{2}$.

\section{The WiLE Mathematica ${ }^{\circledR}$ package}

In this section we introduce the package Mathematica ${ }^{\circledR}$ WiLE and its usage. The purpose of the package is to compute the weak coupling expansion of Wilson loops in $\mathrm{ABJ}(\mathrm{M})$ in terms of Feynman diagrams as in (21) for any contour and at any loop order specified by the user. In the following we present the setup of the package and a detailed manual. 


\subsection{Download and installation}

The package set up is very simple. All the relevant files can be downloaded from the arXiv servers with the source files of this paper ${ }^{9}$. The most updated version of the package can be also downloaded from the GitHub repository at the following address

https://github.com/miciosca/WiLE.

The package WiLE.m can be loaded into any Mathematica ${ }^{\circledR}$ notebook saved in the same directory with the command

$\ln [1]:=$ SetDirectory [NotebookDirectory [] ]

$<$ WiLE.m

or

$\ln [1]:=$ SetDirectory [ NotebookDirectory [] ]

Needs [ "WiLE" "]

It is also possible to install the package in such a way that it is available for any notebook without specify any directory. To do it, select "File", then "Install" and then follow the instructions. After installing, the package can be loaded with the commands above without the function SetDirectory.

\subsection{Manual}

The new available functions can be listed using the command

$\ln [3]:=$ ? WiLE`*

and they are the following

- WiLE: The main function of the package. Given the number and the type of the fields on the Wilson loop and the vertices from the action, the function draws all the possible diagrams with the related integrands;

- WiLEFullorder: It draws diagrams with the related integrands for a given order in the weak coupling expansion of the Wilson loop;

- WiLESimplify: It simplify the integrands.

In the following we briefly describe their applications.

WiLE : When the package is loaded, run the command

$\ln [3]:=$ WiLE

\footnotetext{
${ }^{9}$ In the abstract page of this paper click on "Other formats" and then on "Download source". The downloaded file is a compressed directory without extension that needs to be uncompressed. The package and a Mathematica ${ }^{\circledR}$ notebook containing an explicit example can be found in the "WiLE" directory.
} 
without semicolon at the end. The output will be the following window

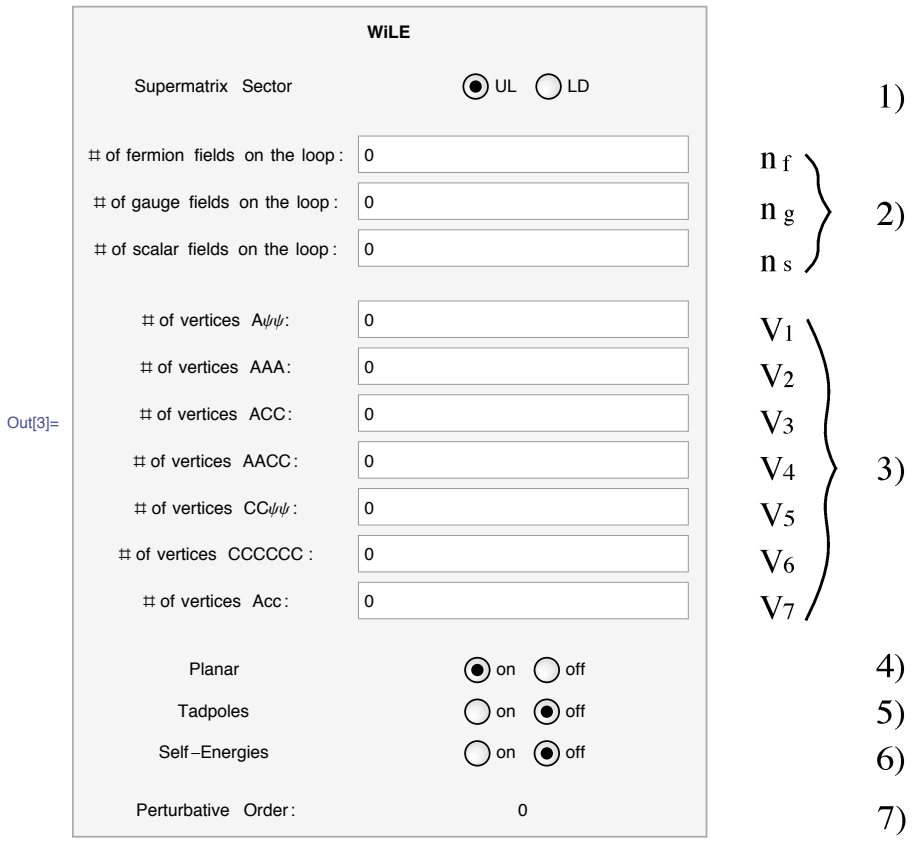

7)

The WiLE output can be computed filling the input fields with a suitable set of initial data and selecting the wanted options and then pressing the usual Shift+Enter. In the following we have listed the instructions for any input line in $(24)^{10}$.

1. Supermatrix Sector: This option allows the user to choose between the upper-left or the lower right blocks of the supermatrix $W[C]$. In other words, choosing the options UL or LR the diagrams in the output will be part of the vev's $\left\langle\mathcal{W}^{\uparrow}[C]\right\rangle$ or $\left\langle\mathcal{W}^{\downarrow}[C]\right\rangle$ respectively (see (15));

2. \# of fermion, gauge and scalar fields on the loop: The user has to specify the number (a positive integer or 0) of the three available fields on the Wilson loop: $n_{f}$ fermion fields $\psi$ or $\bar{\psi}, n_{g}$ gauge fields $A_{\mu}$ or $\hat{A}_{\mu}$ and $n_{s}$ couples of scalar fields $C \bar{C}$ or $\bar{C} C$. The above Supermatrix sector option is the discriminant between the two possibilities for any $n_{i}$. Given the triplet $\left(n_{f}, n_{g}, n_{s}\right)$, the algorithm selects the wanted monomials in the expansion of $\operatorname{Tr}_{\mathbf{N}(\mathbf{M})}\left(W^{\uparrow(\downarrow)}[C]\right)$. To give an example, for the path-ordered expansion of $\operatorname{Tr}_{\mathbf{N}}\left(W^{\uparrow}[C]\right)$ in (16), the triplet $(0,0,0)$ selects the first term $\mathbb{1}_{\mathbf{N}}$, the triplet $(0,1,1)$ selects the $\mathcal{A}_{1} \mathcal{A}_{2}$ monomial in which both the connections can contribute as a gauge field or a couple of scalars (see (4)) but not the same simultaneously and the triplet $(2,1,0)$ selects the last three monomials in which $\mathcal{A}$ contributes as a gauge field. It is clear from the equation (16), that the choice of the triplet $\left(n_{f}, n_{g}, n_{s}\right)$ fixes the number of nested integrals (17). Indeed if the output of WiLE for a given triplet $\left(n_{f}, n_{g}, n_{s}\right)$ is $F\left(n_{f}, n_{g}, n_{s}\right)$ the related integral is

$$
\int_{C} d \tau_{1>2>\ldots>n-1>n} F\left(n_{f}, n_{g}, n_{s}\right) \quad \text { with } \quad n=n_{f}+n_{g}+n_{s}
$$

\footnotetext{
${ }^{10}$ The list numbers are related to the numbers appearing in the panel (24). These labels are added for simplicity and then they are not present in the WiLE input window.
} 
3. \# of vertices: The user has to specify the number (a positive integer or 0 ) of the seven available interaction vertices of the action. More in detail:

- \# of vertices $A \psi \psi$ : It is the number $V_{1}$ of three-gauge-fermion vertices coming from the term $\bar{\psi}^{I} \not D \psi_{I}$ of the matter action $S_{\text {matter }}$ (see (91));

- \# of vertices $A A A$ : It is the number $V_{2}$ of three-gauge vertices coming from the terms $A_{\mu} A_{\nu} A_{\rho}$ and $\hat{A}_{\mu} \hat{A}_{\nu} \hat{A}_{\rho}$ of the Chern-Simons action $S_{\mathrm{CS}}$ (see (89));

- \# of vertices $A C C$ : It is the number $V_{3}$ of three-gluon-scalar vertices coming from the term $D_{\mu} C^{I} D^{\mu} \bar{C}_{I}$ of the matter action $S_{\text {matter }}$ (see (91));

- \# of vertices $A A C C$ : It is the number $V_{4}$ of four-gluon-scalar vertices coming from the term $D_{\mu} C^{I} D^{\mu} \bar{C}_{I}$ of the matter action $S_{\text {matter }}$ (see (91));

- \# of vertices $C C \psi \psi$ : It is the number $V_{5}$ of four-fermion-scalar vertices coming from the Yukawa term $S_{\text {pot }}^{F}$ of the action (see (92));

- \# of vertices $C C C C C C$ : It is the number $V_{6}$ of six-scalar vertices coming from the sextic potential term $S_{\text {pot }}^{B}$ of the action (see (93));

- \# of vertices $A c c$ : It is the number $V_{7}$ of three-gauge-ghost vertices coming from the terms $\partial_{\mu} \bar{c} D^{\mu} c$ and $\partial_{\mu} \overline{\hat{c}} D^{\mu} \hat{c}$ of the gauge-fixing action $S_{\mathrm{gf}}$ (see (90)).

According to our notation, the position of the vertices is denoted by the letter $z_{i}$ with the index $i=1, \ldots, v$ where $v$ is the total number of vertices $v=\sum_{i=1}^{7} V_{i}$. It is clear from the definition (15) that any vertex comes with an integration over $z$ over all the space $\mathbb{R}^{3}$. Then, if the output of WiLE for a given number of vertices is $F\left(V_{1}, V_{2}, V_{3}, V_{4}, V_{5}, V_{6}, V_{7}\right)$ the related integral is

$$
\int d^{3} z_{1} \int d^{3} z_{2} \ldots \int \ldots \int d^{3} z_{v} F\left(V_{1}, V_{2}, V_{3}, V_{4}, V_{5}, V_{6}, V_{7}\right) \quad \text { with } \quad v=\sum_{i=1}^{7} V_{i}
$$

We set the dimension of the above integrals to be $D=3$ but it is possible to keep the dimension arbitrary to dimensional-regularize the integrals (for more details see the explicit example in section 4).

4. Planar: This option allows the user to choose to expand the Wilson loop in the planar 't Hooft limit (23) or not. Since the color structure of a given diagram in general is $M^{s} N^{t}$ with $s, t=0,1, \ldots$, the quantity $s+t$ has to obey to the following bound

$$
0 \leq s+t \leq p-v+1
$$

with $p$ the number of propagators. If the option Planar is on, then the algorithm selects only the planar diagrams, namely the diagrams that saturate the upper bound of (27). Otherwise the output will contain all the possible color structures for any diagram.

5. Tadpoles: This option allows the user to choose if tadpoles diagrams are listed into the output or not. From a topological point of view, tadpoles are identified as diagrams that are not connected anymore if one of the vertices is deleted (here we are considering the 
position on the loop $x_{i}$ as vertex). For instance the last two diagrams in (22) are tadpoles. Their value depends on the regularization scheme: in dimensional regularization is well known that this kind of diagrams vanish then, if this is the selected scheme, it is useful to select the option Tadpoles on off. In general, for a given set of initial data, the number of tadpole diagrams is huge, then selecting off the number of diagrams in the output will be significantly reduced.

6. Self-Energies: This option allows the user to choose if self-energy diagrams are listed into the output or not. From a topological point of view, self-energies are defined as diagrams that are not connected anymore if two edges are cut. From a physical point of view, self-energies are the diagrams in which one or more of the propagators are substituted by a $\ell$-loop corrected propagator with $\ell$ depending on the perturbative order. For instance, at one-loop there are no self-energies (see (22)), at two-loop the self-energies can be computed starting from the one-loop diagrams and dressing them with the one-loop effective propagators and so on. Using this method, the number of loop integrations decrease and then it is useful to select the option Self-Energies on off and reads the self-energies from the dressing of the previous perturbation theory orders. Otherwise, since the $\ell$-loop effective propagators are not known at any $\ell$, the algorithm will print the self-energy diagrams as a explicit $\ell$-loop integrals.

7. Perturbative order: Given the number of the fields on the loop $\left(n_{f}, n_{g}, n_{s}\right)$ and the number of vertices $V_{i}$ with $i=1, \ldots, 7$, it shows in real-time the perturbation theory order $\ell=p-v$ in which the diagrams in the output are included. It is clear from the definition (21) that $\ell$ has to be a positive integer number. If it is not, the message $\notin$ Integers will be displayed.

The output of the function WiLE is a matrix. Any row of the matrix consists of two elements: the first is the diagram drawing and the second is the related integrand. For instance, if one sets the number of fields on the loop as $\left(n_{f}=2, n_{g}=1, n_{s}=1\right)$, the number of vertices as $V_{1}=V_{5}=1$ and the other to zero and the remaining options as in (24), the function will generate a matrix with 24 rows. Since the perturbative order is $\ell=4$, these 24 diagrams contribute to $\frac{a_{4}^{\uparrow}(N, M)}{\kappa^{4}}$ (see (21)) then they are involved in the four-loop computation of $\left\langle\mathcal{W}_{ \pm}[C]\right\rangle$ (see (14)). One of these diagrams for example is the following

$$
\begin{aligned}
& \left\{\frac{1}{\kappa^{4}} 16 \pi^{4} \mathrm{M}^{3} \mathrm{~N}^{2} \eta_{2} \cdot \gamma^{\sigma_{3}} \cdot \gamma^{\mu_{1}} \cdot \gamma^{\sigma_{4}} \cdot \gamma^{\sigma_{2}} \cdot \bar{\eta}_{4}\right. \\
& \mathrm{d}_{\mathbf{x}_{4}}{ }^{\sigma_{2}}\left[\Delta\left[\mathbf{x}_{4}, \mathbf{z}_{2}\right]\right] \mathrm{d}_{\mathbf{z}_{1}}{ }^{\mu_{3}}\left[\Delta\left[\mathbf{z}_{1}, \mathbf{x}_{3}\right]\right] \mathrm{d}_{\mathbf{z}_{1}}{ }^{\sigma_{3}}\left[\Delta\left[\mathbf{z}_{1}, \mathbf{x}_{2}\right]\right] \mathrm{d}_{\mathbf{z}_{2}}{ }^{\sigma_{4}}\left[\Delta\left[\mathbf{z}_{2}, \mathbf{z}_{1}\right]\right] \bmod \left[\dot{\mathbf{x}}_{1}\right] \\
& \bmod \left[\dot{\mathbf{x}}_{2}\right] \bmod \left[\dot{\mathbf{x}}_{4}\right] \\
& \left.\left(2 \overline{\mathbf{n}}_{4} \cdot \mathrm{M}_{1} \cdot \mathrm{n}_{2}-\mathrm{n}_{2} \cdot \overline{\mathbf{n}}_{4} \operatorname{tr}\left[\mathrm{M}_{1}\right]\right) \dot{\mathbf{x}}_{3}{ }^{\mu_{2}} \Delta\left[\mathbf{x}_{1}, \mathbf{z}_{2}\right] \Delta\left[\mathbf{z}_{2}, \mathbf{x}_{1}\right] \epsilon_{\mu_{1} \mu_{2} \mu_{3}}\right\}
\end{aligned}
$$

where the integrand is written using the built-in Mathematica ${ }^{\circledR}$ package Notation. The symbols appearing in the integrand are written in order to be as much as possible similar to the related quantities defined in section 2. The complete set of symbols involved in the output is listed in appendix D. Briefly, the red thick lines represents the Wilson loop, the black lines are fermions 
propagators, the green line is a gauge propagator and the blue lines are scalar propagators. The encircled numbers on top and outside the Wilson loop stand for the positions $x_{i}$ with $i=1, \ldots, 4$ and $z_{j}$ with $j=1,2$ respectively. The loop is oriented counter-clockwise then $\tau_{1}>\tau_{2}>\tau_{3}>\tau_{4}$. It is possible to recover the number and the kind of the integrations in front of this output simply looking at the diagram. Indeed, following the prescription given in (25) and (26) and using the legend in appendix D, we can write

$$
\begin{aligned}
> & =\left(\frac{2 \pi}{\kappa}\right)^{4} M^{3} N^{2} \int_{C} d \tau_{1>2>3>4} \int d^{3} z_{1} d^{3} z_{2}\left(\left|\dot{x}_{1}\right|\left|\dot{x}_{2}\right|\left|\dot{x}_{4}\right|\right. \\
& \times\left[2\left(\bar{n}_{4} \mathcal{M}_{1} n_{2}\right)-\left(n_{2} \bar{n}_{4}\right) \operatorname{tr}\left(\mathcal{M}_{1}\right)\right] \epsilon_{\mu_{1} \mu_{3} \nu_{3}} \dot{x}_{3}^{\nu_{3}}\left(\eta_{2} \gamma^{\sigma_{3}} \gamma^{\mu_{1}} \gamma^{\sigma_{4}} \gamma^{\sigma_{2}} \bar{\eta}_{4}\right) \\
& \left.\times \Delta\left(x_{1}, z_{2}\right)^{2} \partial_{x_{2}}^{\sigma_{3}} \Delta\left(x_{2}, z_{1}\right) \partial_{x_{3}}^{\mu_{3}} \Delta\left(x_{3}, z_{1}\right) \partial_{x_{4}}^{\sigma_{2}} \Delta\left(x_{4}, z_{2}\right) \partial_{z_{1}}^{\sigma_{4}} \Delta\left(z_{1}, z_{2}\right)\right)
\end{aligned}
$$

where the $\partial_{x}^{\mu}=\frac{\partial}{\partial x^{\mu}}$ and $\Delta(x, y)$ is the kinematic part of the propagator defined in (98).

For a given set of initial data, the output of WiLE could be zero or even non existent. In both cases the printed output will be an empty matrix \{\} . The user will be notified by warnings if the output is zero for some very specific reasons. For instance if the number of fields is odd, if all the diagrams in the output are not connected or if the initial data are invalid (i.e. the inputs are not zeroes or positive integers).

WiLEFullorder : When the package is loaded, run the command

$\ln [3]:=$ WiLEFullorder

without semicolon at the end. The output will be the following window

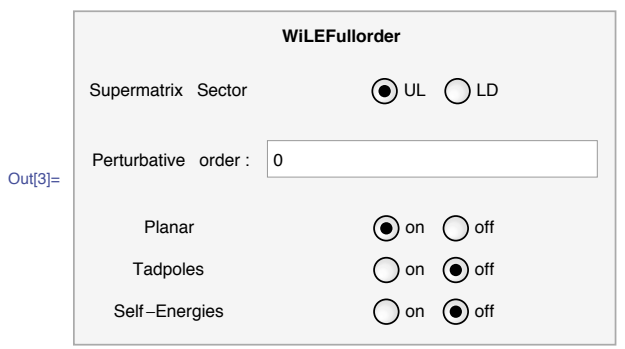

This function prints the complete set of diagrams and integrand for a given perturbative order $\ell$ contributing to $\frac{a_{\ell}^{\uparrow(\downarrow)}(N, M)}{\kappa^{\ell}}$ (see (21)). The input window (29) can be used as the previous one, the only difference is the kind of initial data. Indeed here the user, in addition to the options described in the previous paragraph, needs only to specify the wanted perturbative order $\ell$. As the function WiLE, the output is a matrix with one diagram and its related integrand for any rows. The output for $\ell=0$ is clearly the gauge group rank depending on the choice of the supermatrix sector. The first non-trivial order is given by setting the perturbative order $\ell=1$ as we have seen schematically in (22). In that case the output is 


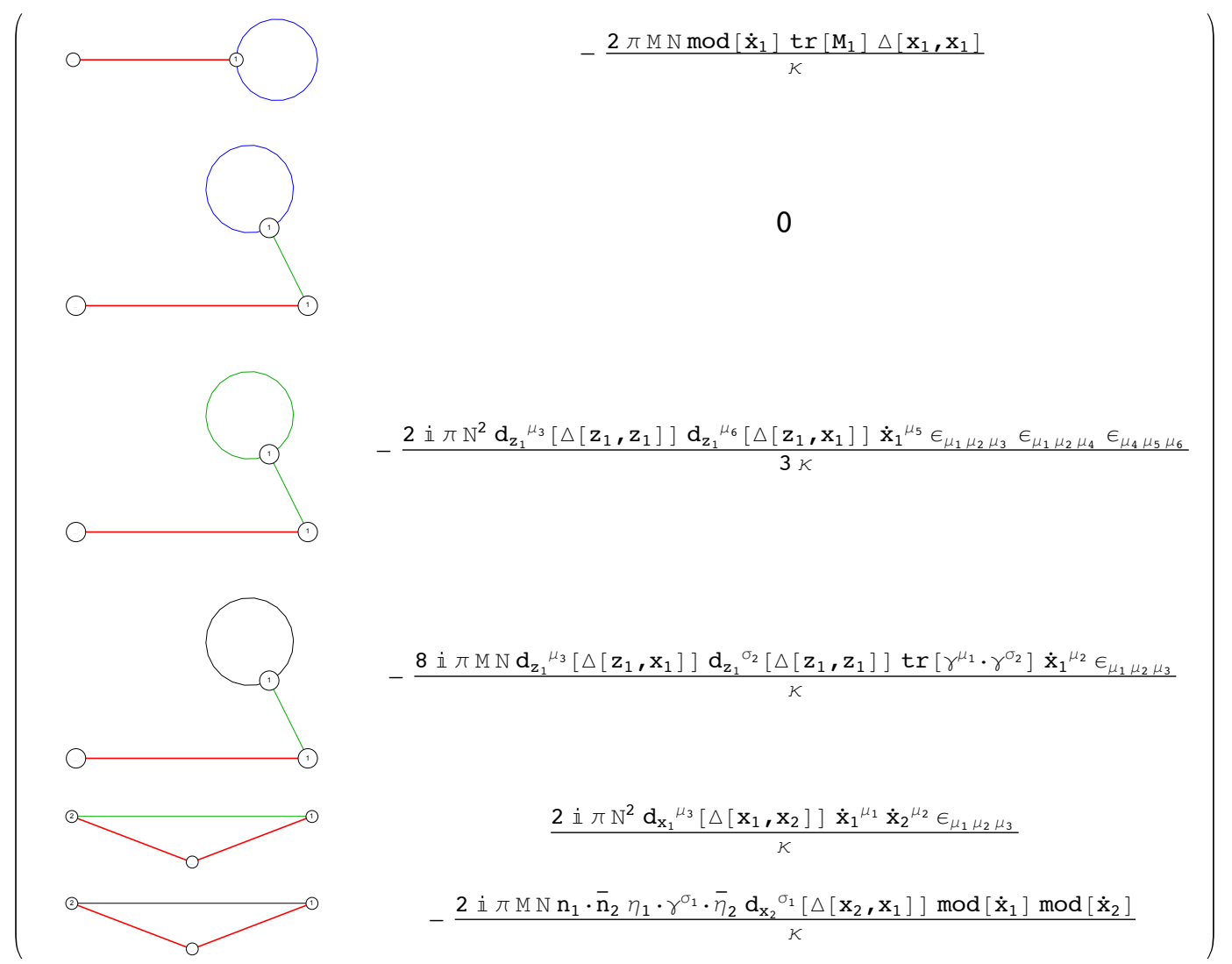

where we have used the built-in command MatrixForm for clearness purposes. The output above is computed selecting the most general options (Planar=off, Tadpoles=on and Self-enegies=on) then it contains all the possible diagrams contributing to $\frac{a_{1}^{\uparrow}(N, M)}{\kappa}$. As we have seen from the equation (22), we have three different diagram topologies. Comparing this result with the topologies in (22), and adding the needed integrals in front of the integrands following the prescriptions given in (25) and (26), we have
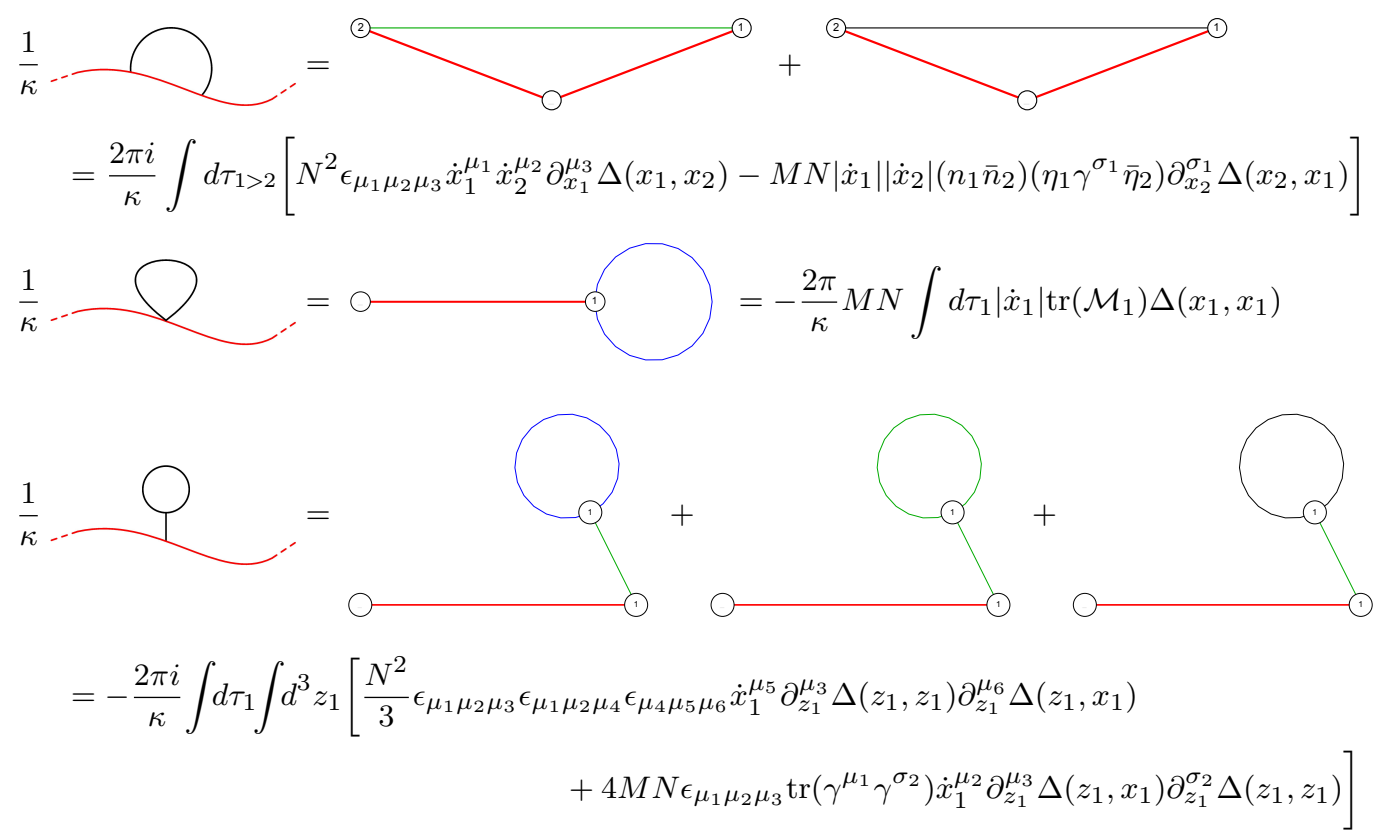

where we have used the legend in appendix D. 
If the input data are valid (i.e. Perturbative order zero or a positive integer), the output of WiLEFullorder cannot be zero. The execution speed of this function decreases quickly at the raising of the perturbative order because the number of the diagrams factorially (or exponentially in the planar case) grows ${ }^{11}$. In the following table we show the number of diagrams for a given perturbative order $\ell$ varying the options available in the input window (29)

\begin{tabular}{lcccc} 
& $\ell=0$ & $\ell=1$ & $\ell=2$ & $\ell=3$ \\
\hline$\{$ on,off,off $\}$ & 0 & 2 & 15 & 219 \\
$\{$ off,off,off $\}$ & 0 & 2 & 16 & 276 \\
off,off,on $\}$ & 0 & 2 & 24 & 826 \\
\{off,on,on $\}$ & 0 & 6 & 92 & 2583
\end{tabular}

where the vectors in the first column refer to \{Panar, Tadpoles, Self-Energies $\}$.

WiLESimplify : This command can be used as the Mathematica ${ }^{\circledR}$ built-in function Simplify. The main purpose of the function WiLESimplify is to simplify expressions with a large number of $\epsilon$-tensors (both with Lorentz and R-symmetry indices). It is useful also for simplify very long expressions.

\section{Example: The 3-loop ladder diagrams of a Wilson line with a cusp}

Ladder diagrams are usually the most simple class of diagrams in the perturbative series because they do not involve interaction vertices. At one-loop for instance, the total contribution to the expectation value of an arbitrary Wilson loop is given only by the ladder diagrams $(30)^{12}$, but at higher loops diagrams with interaction vertices start to contribute. In the literature there are many examples in which ladder diagrams play a fundamental role in the computation of some observables of interest. For instance the expectation value of Wilson loops on the sphere $S^{2}$ in $\mathcal{N}=4 \mathrm{SYM}$ is given only by the resummation of ladder diagrams [9]. The same for correlation functions of Wilson loops and local operators on $S^{2}$ [19]. In [52] the authors identified a scaling limit in which only ladder diagrams contribute to the expectation value of Wilson loop with a cusp. These ladders can be resummed solving a Schrodinger problem. It was also done in $\mathrm{ABJ}(\mathrm{M})$ theory [39]. In this case the related Schrodinger problem is exactly solvable and it provides the solution for $\mathcal{W}_{ \pm}$in a closed exponential form. The expectation value of the cusped Wilson line is divergent and its divergence is related to the generalized cusp anomalous dimension $\Gamma_{\text {cusp }}(\lambda, \theta, \varphi)$. At $\theta= \pm \varphi$ the cusped Wilson line becomes BPS and then

\footnotetext{
${ }^{11}$ More quantitatively with a laptop with $2.6 \mathrm{GHz}$ Intel Core $\mathrm{i} 5$ processor and $8 \mathrm{~Gb}$ or DDR3 RAM, the $\ell=0,1$ cases are computed almost instantly, $\ell=2$ in the order of seconds and $\ell=3$ in the order of minutes (the most general case needs more than 1 hour). Very high-loop cases are hard to achieve using WiLEFullorder, indeed we did a test for the $\ell=4$ case and the planar case needs more or less 1 day to be computed. Anyway at higher loop is easier to use the command WiLE for the single graph topology and parallelize the computation in order to compute many topologies at the same time. This option is not available in the current version of the package but we will implement it in a future release.

${ }^{12}$ If we neglect the tadpoles (31) and (32).
} 
its anomalous dimension vanishes. Around these BPS points $\Gamma_{\text {cusp }}$ can be expanded and the first non-trivial order is given by the Bremsstrahlung function. This function was recently studied up to three-loops in [41]. In order to compute this observable, the three-loop ladder diagrams was computed using the HQET formalism.

In this section we want to present an explicit example in which the package WiLE is useful giving also a three-loop perturbative check of the exponentiation of ladders [39] and the ladder diagrams computed with the HQET formalism in [41].

\subsection{The generalized cusped Wilson line}

Consider two Wilson lines $C_{1}$ and $C_{2}$ on $\mathbb{R}^{3}$ intersecting in the origin forming the curve $C=$ $C_{1} \cup C_{2}$ as in Figure 1. The angle between the two lines is $\pi-\varphi$ such that at $\varphi=0$ the contour becomes a straight line without the cusp. The parametrization of the contour is

$$
x_{\mu}=\left\{0, \tau \cos \frac{\varphi}{2},|\tau| \sin \frac{\varphi}{2}\right\} \quad-L \geq \tau \leq L
$$

with $L$ an IR cut-off shielding the infinite length of the lines.

The fermionic Wilson loop operators lying on this contour are defined in (12). In general one can choose different R-symmetry couplings for the two edges. In other words, one can consider an angle $\theta$ that denotes the angular separation of the two Wilson lines in the internal R-symmetry space (or $\mathbb{C P}^{3}$ from the string side). The fermionic couplings have the factorized structure presented in (6) and they can be read from (11) setting $\alpha=0$. Since the reduced vector couplings $n$ and $\bar{n}$ are unconstrained, they can be chosen to be function of the $\theta$ angle such that

$$
\left(n_{1} \bar{n}_{2}\right)=\cos \frac{\theta}{2} \quad \text { and } \quad\left(n_{1} \bar{n}_{1}\right)=\left(n_{2} \bar{n}_{2}\right)=1
$$

where the indices 1 and 2 specify the edges of the cusp. In particular for the first edge they are given by

$$
n_{1 I}=\left(\begin{array}{llll}
\cos \frac{\theta}{4} & \sin \frac{\theta}{4} & 0 & 0
\end{array}\right) \quad \eta_{1}^{\alpha}=\left(\begin{array}{ll}
e^{-i \frac{\varphi}{4}} & e^{i \frac{\varphi}{4}}
\end{array}\right) \quad \bar{n}_{1}^{I}=\left(\begin{array}{c}
\cos \frac{\theta}{4} \\
\sin \frac{\theta}{4} \\
0 \\
0
\end{array}\right) \quad \bar{\eta}_{1 \alpha}=i\left(\begin{array}{c}
e^{i \frac{\varphi}{4}} \\
e^{-i \frac{\varphi}{4}}
\end{array}\right)
$$

and for the second edge are given by

$$
n_{2 I}=\left(\begin{array}{cccc}
\cos \frac{\theta}{4} & -\sin \frac{\theta}{4} & 0 & 0
\end{array}\right) \quad \eta_{2}^{\alpha}=\left(\begin{array}{ll}
e^{i \frac{\varphi}{4}} & e^{-i \frac{\varphi}{4}}
\end{array}\right) \quad \bar{n}_{2}^{I}=\left(\begin{array}{c}
\cos \frac{\theta}{4} \\
-\sin \frac{\theta}{4} \\
0 \\
0
\end{array}\right) \quad \bar{\eta}_{2 \alpha}=i\left(\begin{array}{c}
e^{-i \frac{\varphi}{4}} \\
e^{i \frac{\varphi}{4}}
\end{array}\right)
$$

From the relation (8) one can read also the scalar couplings $\mathcal{M}$ and $\hat{\mathcal{M}}$. Indeed using the 


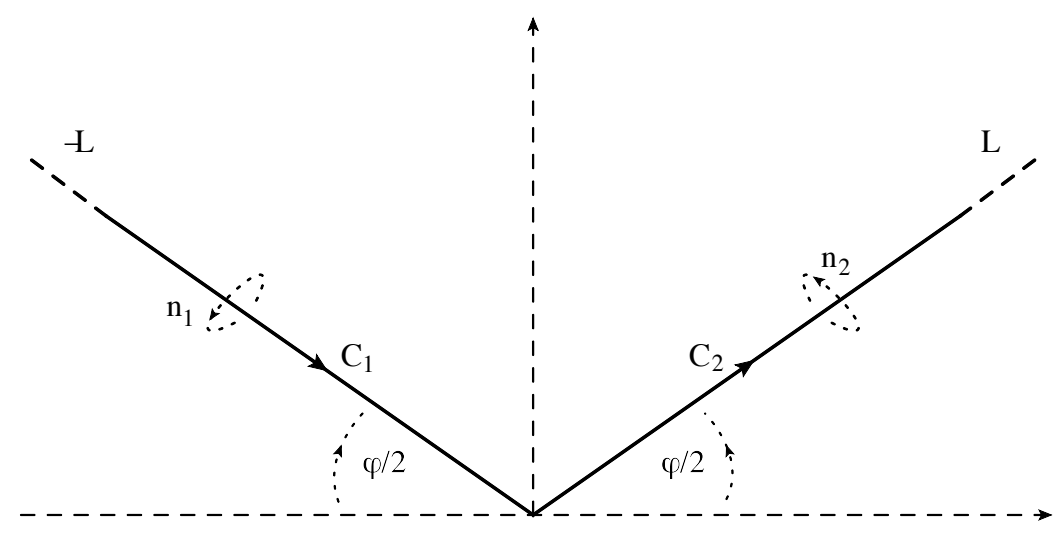

Figure 1: The planar Euclidean cusp with angular extension given by $\pi-\varphi$.

definitions (35) and (36) we have

$\mathcal{M}_{1 J}^{I}=\hat{\mathcal{M}}_{1 J}^{I}=\left(\begin{array}{cccc}-\cos \frac{\theta}{2} & -\sin \frac{\theta}{2} & 0 & 0 \\ -\sin \frac{\theta}{2} & \cos \frac{\theta}{2} & 0 & 0 \\ 0 & 0 & 1 & 0 \\ 0 & 0 & 0 & 1\end{array}\right) \quad$ and $\quad \mathcal{M}_{2 J}^{I}=\hat{\mathcal{M}}_{2 J}^{I}=\left(\begin{array}{cccc}-\cos \frac{\theta}{2} & \sin \frac{\theta}{2} & 0 & 0 \\ \sin \frac{\theta}{2} & \cos \frac{\theta}{2} & 0 & 0 \\ 0 & 0 & 1 & 0 \\ 0 & 0 & 0 & 1\end{array}\right)$

In general the cusped Wilson line is not a BPS operator but if $\theta= \pm \varphi$, it preserves two supersymmetries. Then in this configuration the operator is globally $1 / 6$ BPS.

The bosonic Wilson loops $\mathcal{W}_{B}$ and $\hat{\mathcal{W}}_{B}$ lying on the contour represented in figure 1 have the following bosonic couplings

$M_{1 J}^{I}=\hat{M}_{1 J}^{I}=\left(\begin{array}{cccc}-\cos \frac{\theta}{2} & -\sin \frac{\theta}{2} & 0 & 0 \\ -\sin \frac{\theta}{2} & \cos \frac{\theta}{2} & 0 & 0 \\ 0 & 0 & -1 & 0 \\ 0 & 0 & 0 & 1\end{array}\right) \quad$ and $\quad M_{2 J}^{I}=\hat{M}_{2 J} I=\left(\begin{array}{cccc}-\cos \frac{\theta}{2} & \sin \frac{\theta}{2} & 0 & 0 \\ \sin \frac{\theta}{2} & \cos \frac{\theta}{2} & 0 & 0 \\ 0 & 0 & -1 & 0 \\ 0 & 0 & 0 & 1\end{array}\right)$

Also in this case the cusped bosonic Wilson line is not a BPS operator but if $\theta= \pm \varphi$, it preserves one supersymmetry. Then in this configuration the operator is globally $1 / 12$ BPS.

\subsection{The perturbative computation}

The evaluation of ladder diagrams of the cusped Wilson loop obviously encounters UV divergences which originate when the propagators endpoints coincide. To tame these divergences we have to use dimensional regularization. In particular we will follow the DRED scheme in which the dimension of space-time is set to $d=3-2 \epsilon$ but the dimension of $\epsilon_{\mu \nu \rho}$ tensors and the Dirac algebra remains three. We have to introduce a mass scale $\mu^{2 \epsilon}$ that keeps the action dimensionless breaking explicitly the conformal invariance. However, because of the underlying conformal invariance, the mass scale $\mu$ with the IR cut-off $L$ introduced in (33) forms the combination $(\mu L)^{2 \ell \epsilon}$ that can be always scaled away in any Feynman integral at $\ell$-loops.

Ladder diagrams of a fermionic Wilson loop in $\mathrm{ABJ}(\mathrm{M})$ involve three kind of propagators: the 
fermionic propagator, the gauge propagator and a couple of scalar propagators sharing the same starting and ending points (they appear on the loop always as the bilinears $C \bar{C}$ or $\bar{C} C$ ). Ladder diagrams of bosonic Wilson loops instead do not involve fermionic propagators. When the contour of the loop is parametrized as a two-dimensional curve (as for instance in our case (33)), ladder diagrams involving at least one gauge propagator vanish. Indeed, the gauge propagator is proportional to the following combination

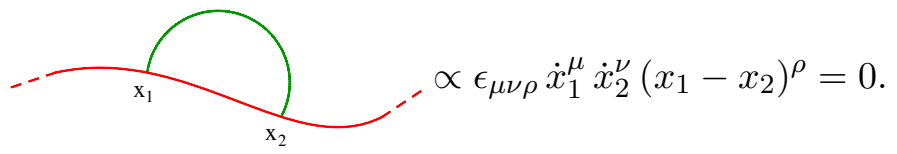

that is clearly zero for a two-dimensional curve. As a consequence of this observation, since at three-loops operators $\mathcal{W}_{A}$ and $\hat{\mathcal{W}}_{A}$ contain only gauge propagators and operators $\mathcal{W}_{B}$ and $\hat{\mathcal{W}}_{B}$ contain at least one gauge propagator, the ladder part of their expectation values vanishes. In general this is true for any odd perturbative order $\ell$, then

$$
\begin{array}{ll}
\left\langle\mathcal{W}_{A}\left[C_{2 d}\right]\right\rangle_{\text {ladder }}^{(\ell)}=\left\langle\hat{\mathcal{W}}_{A}\left[C_{2 d}\right]\right\rangle_{\text {ladder }}^{(\ell)}=0 & \ell \in \operatorname{Odd}\left(\mathbb{Z}^{+}\right) \\
\left\langle\mathcal{W}_{B}\left[C_{2 d}\right]\right\rangle_{\text {ladder }}^{(\ell)}=\left\langle\hat{\mathcal{W}}_{B}\left[C_{2 d}\right]\right\rangle_{\text {ladder }}^{(\ell)}=0 & \ell \in \operatorname{Odd}\left(\mathbb{Z}^{+}\right) .
\end{array}
$$

where $C_{2 d}$ denotes an arbitrary two-dimensional contour.

For the fermionic Wilson loop the story is different. Indeed, at any loop order there is at least a class of ladder diagrams without gauge propagators (see for instance the one-loop expansion (30)). In particular, at three-loops the ladder diagrams are given by the following fermionic monomial

$$
-\left(\frac{2 \pi}{\kappa}\right)^{3}\left|\dot{x}_{1}\right|\left|\dot{x}_{2}\left\|\dot{x}_{3}\right\| \dot{x}_{4}\right|\left|\dot{x}_{5}\right|\left|\dot{x}_{6}\right| \operatorname{Tr}_{N}\left[(\eta \bar{\psi})_{1}(\psi \bar{\eta})_{2}(\eta \bar{\psi})_{3}(\psi \bar{\eta})_{4}(\eta \bar{\psi})_{5}(\psi \bar{\eta})_{6}\right]
$$

and the following mixed bosonic-fermionic monomials

$$
\begin{aligned}
-\left(\frac{2 \pi}{\kappa}\right)^{3}\left|\dot{x}_{1}\left\|\dot{x}_{2}|| \dot{x}_{3}\right\|\right. & \dot{x}_{4} \mid \operatorname{Tr}_{N}\left[(\eta \bar{\psi})_{1}(\psi \bar{\eta})_{2}(\mathcal{M} C \bar{C})_{3}(\mathcal{M} C \bar{C})_{4}+(\mathcal{M} C \bar{C})_{1}(\eta \bar{\psi})_{2}(\psi \bar{\eta})_{3}(\mathcal{M} C \bar{C})_{4}\right. \\
& +(\eta \bar{\psi})_{1}(\hat{\mathcal{M}} \bar{C} C)_{2}(\hat{\mathcal{M}} \bar{C} C)_{3}(\psi \bar{\eta})_{4}+(\mathcal{M} C \bar{C})_{1}(\mathcal{M} C \bar{C})_{2}(\eta \bar{\psi})_{3}(\psi \bar{\eta})_{4} \\
& \left.+(\eta \bar{\psi})_{1}(\hat{\mathcal{M}} \bar{C} C)_{2}(\psi \bar{\eta})_{3}(\mathcal{M} C \bar{C})_{4}+(\mathcal{M} C \bar{C})_{1}(\eta \bar{\psi})_{2}(\hat{\mathcal{M}} \bar{C} C)_{3}(\psi \bar{\eta})_{4}\right]
\end{aligned}
$$

of the expansion (16) where the short hand notation $(\mathcal{M} C \bar{C})_{i}$ and $(\hat{\mathcal{M}} \bar{C} C)_{i}$ stands for $\left(\mathcal{M}_{i}\right)_{J}{ }^{I} C_{I}\left(x_{i}\right) \bar{C}^{J}\left(x_{i}\right)$ and $\left(\hat{\mathcal{M}}_{i}\right)_{J}^{I} \bar{C}^{J}\left(x_{i}\right) C_{I}\left(x_{i}\right)$ respectively. Notice that we are focusing our attention on the upperleft $N \times N$ block of the supermatrix (5) in order to compute $\left\langle\mathcal{W}^{\uparrow}\right\rangle_{\text {ladder }}^{(3)}$. The expectation value $\left\langle\mathcal{W}_{ \pm}\right\rangle_{\text {ladder }}^{(3)}$ can be computed using the relation (18). We are also restricting the computation in the large $N$ and $M$ limit (23) in which only the planar diagrams survive. For this motivation we do not consider some non-planar Wick contractions of the monomial (41) and the last two monomials of (42). Finally, we will restrict our computation for $\phi=0$. In other words we will compute ladder diagrams for an Euclidean Wilson line with a cusp in the internal R-symmetry 
space. It is also useful to introduce the deformed coupling

$$
g(\epsilon) \equiv \frac{1}{\kappa} \frac{\Gamma\left(\frac{1}{2}-\epsilon\right)}{\pi^{\frac{1}{2}-\epsilon}}(\mu L)^{2 \epsilon}
$$

to simplify further the results.

In the following we will present some example of fermionic and bosonic ladder computation. The complete and detailed step-by-step evaluation of all the diagrams can be found in the Mathematica ${ }^{\circledR}$ notebook "Ladders.nb" attached to the source files of this paper. The notebook needs the package HypExp [53] for the Hypergeometric function expansions. It takes more or less 15 minutes to run. However all the computation outputs are printed then they can be consulted without running the entire notebook.

\section{Fermionic ladder diagrams}

This class of diagrams can be computed performing the planar Wick contractions of the fermionic monomial (41). In order to do it using the package WiLE we have to set the initial data to

$$
\left(n_{f}=6, n_{g}=0, n_{s}=0\right) \quad \text { and } \quad V_{i}=0 \quad \text { with } \quad i=1,2, \ldots, 7
$$

We are focusing on the upper-left part of the supermatrix then we have to select UL for the option Supermatrix Sector and, since we want to compute diagrams in the 't Hooft coupling, we have to select on for the option Planar. The remaining available options Tadpoles and Self-Energies are clearly irrelevant for ladder diagrams, then we can select off (the algorithm is slightly quicker choosing this option). Given all the initial data the WiLE output is the following 


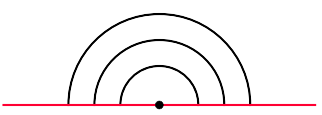

(a) $L_{1}^{1}$

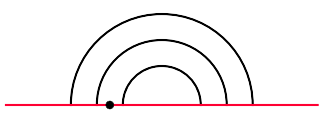

(b) $L_{1}^{2}$

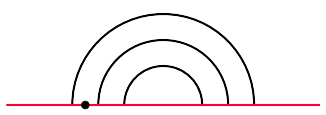

(c) $L_{1}^{3}$

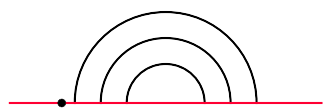

(d) $L_{1}^{4}$

Figure 2: Fermionic ladder diagrams contributing to the diagram $L_{1}$.

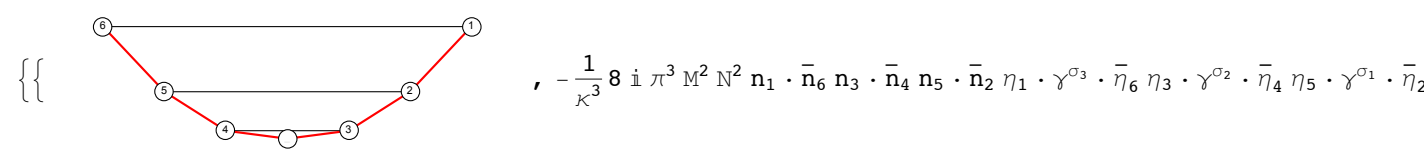

$\left.\mathrm{d}_{\mathbf{x}_{2}}{ }^{\sigma_{1}}\left[\Delta\left[\mathrm{x}_{2}, \mathbf{x}_{5}\right]\right] \mathrm{d}_{\mathbf{x}_{4}}{ }^{\sigma_{2}}\left[\Delta\left[\mathbf{x}_{4}, \mathbf{x}_{3}\right]\right] \mathrm{d}_{\mathbf{x}_{6}}{ }^{\sigma_{3}}\left[\Delta\left[\mathbf{x}_{6}, \mathbf{x}_{1}\right]\right] \bmod \left[\dot{\mathbf{x}}_{1}\right] \bmod \left[\dot{\mathbf{x}}_{2}\right] \bmod \left[\dot{\mathbf{x}}_{3}\right] \bmod \left[\dot{\mathbf{x}}_{4}\right] \bmod \left[\dot{\mathbf{x}}_{5}\right] \bmod \left[\dot{\mathbf{x}}_{6}\right]\right\}$,

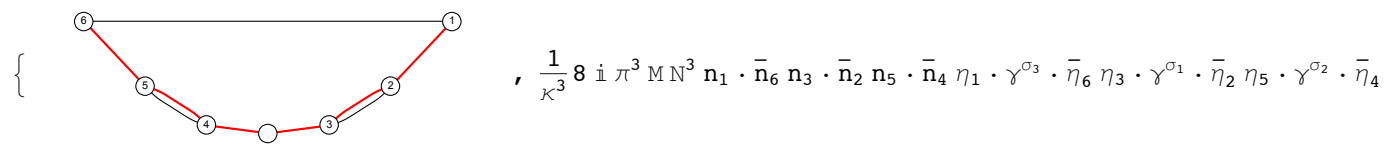

$\left.\mathrm{d}_{\mathbf{x}_{2}}{ }^{\sigma_{1}}\left[\Delta\left[\mathrm{x}_{2}, \mathrm{x}_{3}\right]\right] \mathrm{d}_{\mathbf{x}_{4}}{ }^{\sigma_{2}}\left[\Delta\left[\mathbf{x}_{4}, \mathbf{x}_{5}\right]\right] \mathrm{d}_{\mathbf{x}_{6}}{ }^{\sigma_{3}}\left[\Delta\left[\mathbf{x}_{6}, \mathbf{x}_{1}\right]\right] \bmod \left[\dot{\mathbf{x}}_{1}\right] \bmod \left[\dot{\mathbf{x}}_{2}\right] \bmod \left[\dot{\mathbf{x}}_{3}\right] \bmod \left[\dot{\mathbf{x}}_{4}\right] \bmod \left[\dot{\mathbf{x}}_{5}\right] \bmod \left[\dot{\mathbf{x}}_{6}\right]\right\}$,

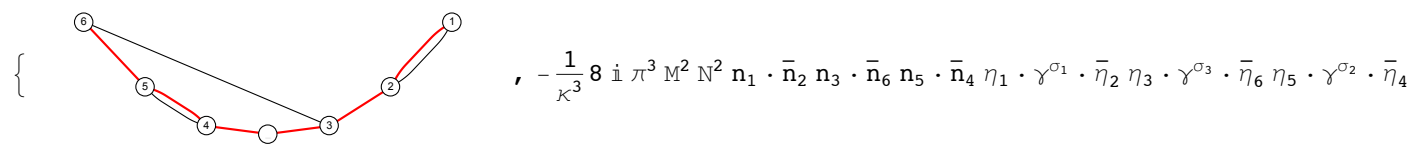

$\left.\mathrm{d}_{\mathbf{x}_{2}}{ }^{\sigma_{1}}\left[\Delta\left[\mathrm{x}_{2}, \mathbf{x}_{1}\right]\right] \mathrm{d}_{\mathbf{x}_{4}}{ }^{\sigma_{2}}\left[\Delta\left[\mathbf{x}_{4}, \mathbf{x}_{5}\right]\right] \mathrm{d}_{\mathbf{x}_{6}}{ }^{\sigma_{3}}\left[\Delta\left[\mathrm{x}_{6}, \mathbf{x}_{3}\right]\right] \bmod \left[\dot{\mathbf{x}}_{1}\right] \bmod \left[\dot{\mathbf{x}}_{2}\right] \bmod \left[\dot{\mathbf{x}}_{3}\right] \bmod \left[\dot{\mathbf{x}}_{4}\right] \bmod \left[\dot{\mathbf{x}}_{5}\right] \bmod \left[\dot{\mathbf{x}}_{6}\right]\right\}$,

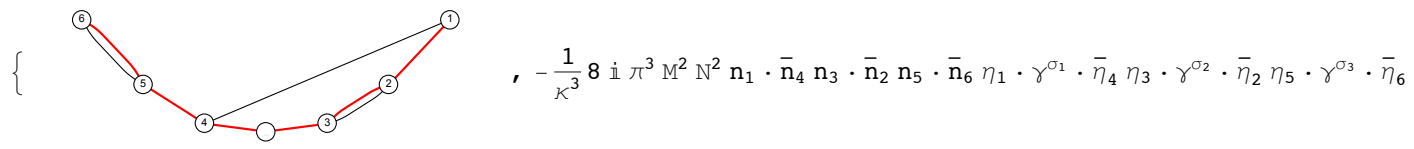

$\left.\mathrm{d}_{\mathrm{x}_{2}}{ }^{\sigma_{2}}\left[\Delta\left[\mathrm{x}_{2}, \mathrm{x}_{3}\right]\right] \mathrm{d}_{\mathbf{x}_{4}}{ }^{\sigma_{1}}\left[\Delta\left[\mathrm{x}_{4}, \mathrm{x}_{1}\right]\right] \mathrm{d}_{\mathbf{x}_{6}}{ }^{\sigma_{3}}\left[\Delta\left[\mathrm{x}_{6}, \mathrm{x}_{5}\right]\right] \bmod \left[\dot{\mathbf{x}}_{1}\right] \bmod \left[\dot{\mathbf{x}}_{2}\right] \bmod \left[\dot{\mathbf{x}}_{3}\right] \bmod \left[\dot{\mathbf{x}}_{4}\right] \bmod \left[\dot{\mathbf{x}}_{5}\right] \bmod \left[\dot{\mathbf{x}}_{6}\right]\right\}$,

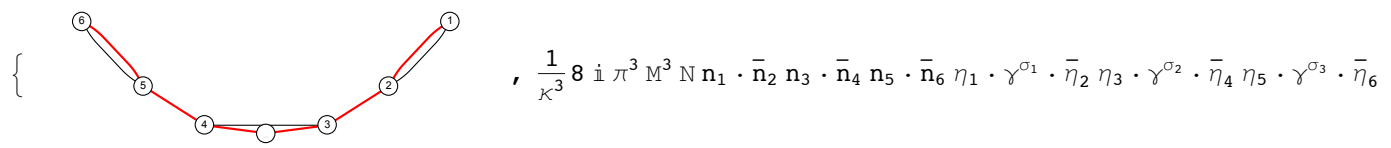

$\left.\left.\mathrm{d}_{\mathbf{x}_{2}}{ }^{\sigma_{1}}\left[\Delta\left[\mathbf{x}_{2}, \mathbf{x}_{1}\right]\right] \mathrm{d}_{\mathbf{x}_{4}}{ }^{\sigma_{2}}\left[\Delta\left[\mathbf{x}_{4}, \mathbf{x}_{3}\right]\right] \mathrm{d}_{\mathbf{x}_{6}}{ }^{\sigma_{3}}\left[\Delta\left[\mathbf{x}_{6}, \mathbf{x}_{5}\right]\right] \bmod \left[\dot{\mathbf{x}}_{1}\right] \bmod \left[\dot{\mathbf{x}}_{2}\right] \bmod \left[\dot{\mathbf{x}}_{3}\right] \bmod \left[\dot{\mathbf{x}}_{4}\right] \bmod \left[\dot{\mathbf{x}}_{5}\right] \bmod \left[\dot{\mathbf{x}}_{6}\right]\right\}\right\}$

In order to simplify the following discussion we name the diagrams above $L_{i}^{\uparrow} i=1, \ldots, 5$ where the index $i$ corresponds to the raws of the WiLE output. Since the contour is the union of two curve $C_{1}$ and $C_{2}$ and the couplings to the matter are different on the two edges (see (35), (36) and (37)), we have to split the region of integration for each diagram in seven sectors corresponding to all the possible positions of the origin. Luckily we do not have to compute all of them. In fact graphs which are related by a reflection with respect to the axis passing through the origin and orthogonal to the Wilson line yield the same result. Indeed using this property the number of unique subdiagrams for any graph $L_{i}^{\uparrow}$ decreases and we have also

$$
L_{4}^{\uparrow}=L_{3}^{\uparrow} .
$$

We will compute in detail the diagram $L_{1}$ as an example.

Following the prescriptions of section 3 and the legend of appendix $\mathrm{D}$, the diagram $L_{1}$ is given 
by

$$
\begin{array}{r}
L_{1}^{\uparrow}=-i\left(\frac{2 \pi}{\kappa}\right)^{3} M^{2} N^{2} \int_{-L}^{L} d \tau_{1>\ldots>6}\left[\left|\dot{x}_{1}\right|\left|\dot{x}_{2}\right|\left|\dot{x}_{3}\right|\left|\dot{x}_{4}\right|\left|\dot{x}_{5}\right|\left|\dot{x}_{6}\right|\left(n_{1} \bar{n}_{6}\right)\left(\eta_{1} \gamma^{\mu} \bar{\eta}_{6}\right) \partial_{x_{6}}^{\mu} \Delta\left(x_{6}, x_{1}\right)\right. \\
\left.\times\left(n_{3} \bar{n}_{4}\right)\left(\eta_{3} \gamma^{\nu} \bar{\eta}_{4}\right) \partial_{x_{4}}^{\nu} \Delta\left(x_{4}, x_{3}\right)\left(n_{5} \bar{n}_{2}\right)\left(\eta_{5} \gamma^{\rho} \bar{\eta}_{2}\right) \partial_{x_{2}}^{\rho} \Delta\left(x_{2}, x_{5}\right)\right]
\end{array}
$$

Because of the reflection property of the diagrams, we have to compute the integral (46) only for the integration regions depicted in Figure 2, namely

$$
\begin{array}{ll}
L_{1}^{1} & \left(-L<\tau_{6}<\tau_{5}<\tau_{4}<0\right) \in C_{1} \cup\left(0<\tau_{3}<\tau_{2}<\tau_{1}<L\right) \in C_{2} \\
L_{1}^{2} & \left(-L<\tau_{6}<\tau_{5}<0\right) \in C_{1} \cup\left(0<\tau_{4}<\tau_{3}<\tau_{2}<\tau_{1}<L\right) \in C_{2} \\
L_{1}^{3} & \left(-L<\tau_{6}<0\right) \in C_{1} \cup\left(0<\tau_{5}<\tau_{4}<\tau_{3}<\tau_{2}<\tau_{1}<L\right) \in C_{2} \\
L_{1}^{4} & \left(0<\tau_{6}<\tau_{5}<\tau_{4}<\tau_{3}<\tau_{2}<\tau_{1}<L\right) \in C_{2}
\end{array}
$$

then the total result can be computed using the following formula

$$
L_{1}^{\uparrow}=L_{1}^{1}+2\left(L_{1}^{2}+L_{1}^{3}+L_{1}^{4}\right)
$$

Consider the diagram $L_{1}^{1}$, we substitute $n \bar{n}$, the bilinears $\eta \gamma \bar{\eta}$ and the propagators $\Delta$ with their values on the contour (47) using the the formulas in appendix B, the definitions (35), (36) and (36) and the parametrization (33). Then the integral (46) becomes

$$
L_{1}^{1}=g(\epsilon)^{3} M^{2} N^{2} \cos ^{3} \frac{\theta}{2} \frac{(1-2 \epsilon)^{3}}{2^{6 \epsilon}} \int_{0}^{1} d \tau_{1>2>3} \int_{0}^{1} d \tau_{6>5>4} \frac{1}{\left[\left(\tau_{3}+\tau_{4}\right)^{2}\left(\tau_{2}+\tau_{5}\right)^{2}\left(\tau_{1}+\tau_{6}\right)^{2}\right]^{1-2 \epsilon}}
$$

where we have rescaled the variables with the dimensional cut-off $L$ as $\tau \rightarrow-L \tilde{\tau}$ on $C_{1}$ and $\tau \rightarrow L \tilde{\tau}$ on $C_{2}$. Then for simplicity we have renamed $\tilde{\tau}$ as $\tau$. Now the integral (52) can be easily computed and it is given by

$$
\begin{aligned}
L_{1}^{1}= & \frac{g(\epsilon)^{3} M^{2} N^{2} \cos ^{3} \frac{\theta}{2}}{3 \times 2^{4+6 \epsilon} \epsilon^{3}(1+2 \epsilon)(1-4 \epsilon)(1-6 \epsilon)}\left[4\left(4 \epsilon\left(2+\epsilon-8 \epsilon^{2}\right)-1\right)-64^{\epsilon}(2 \epsilon+1)(1-2 \epsilon)^{2}\right. \\
& -3 \times 4^{\epsilon+1} \epsilon(2 \epsilon+1)(6 \epsilon-1)-12 \epsilon(1-6 \epsilon)\left(2^{4 \epsilon+1}(4 \epsilon-1){ }_{2} F_{1}(1,4 \epsilon+1 ; 2(\epsilon+1) ;-1)\right. \\
& \left.+(2 \epsilon+1){ }_{2} F_{1}(-2 \epsilon, 4 \epsilon ; 4 \epsilon+1 ;-1)-4 \epsilon_{2} F_{1}(1-4 \epsilon, 2 \epsilon+1 ; 2(\epsilon+1) ;-1)\right) \\
& -12 \epsilon(1-2 \epsilon)(1-6 \epsilon)\left(16^{\epsilon}{ }_{3} F_{2}(1,1-4 \epsilon, 2-2 \epsilon ; 2-4 \epsilon, 2 \epsilon+2 ; 1 / 2)\right. \\
& \left.\left.-2{ }_{3} F_{2}(1,1-4 \epsilon, 2-2 \epsilon ; 2-4 \epsilon, 2 \epsilon+2 ; 1)\right)\right]
\end{aligned}
$$

We repeat the same steps fo the other three diagrams. The diagram $L_{1}^{2}$ has to be computed 
along the contour (48), then the integral (46) becomes

$$
\begin{aligned}
L_{1}^{2}= & g(\epsilon)^{3} M^{2} N^{2} \cos ^{2} \frac{\theta}{2} \frac{(1-2 \epsilon)^{3}}{2^{6 \epsilon}} \int_{0}^{1} d \tau_{1>2>3>4} \int_{0}^{1} d \tau_{6>5} \frac{1}{\left[\left(\tau_{3}-\tau_{4}\right)^{2}\left(\tau_{2}+\tau_{5}\right)^{2}\left(\tau_{1}+\tau_{6}\right)^{2}\right]^{1-2 \epsilon}} \\
= & \frac{g(\epsilon)^{3} M^{2} N^{2} \cos ^{2} \frac{\theta}{2}}{3 \times 2^{4+6 \epsilon} \epsilon^{3}(1+2 \epsilon)(1-4 \epsilon)}\left[2-8 \epsilon^{2}-24 \epsilon^{2}{ }_{2} F_{1}(1-4 \epsilon, 2 \epsilon+1 ; 2(\epsilon+1) ;-1)\right. \\
& -3(1-4 \epsilon)\left((2 \epsilon+1){ }_{2} F_{1}(-2 \epsilon, 4 \epsilon ; 4 \epsilon+1 ;-1)+4^{\epsilon}\left(4 \epsilon_{2} F_{1}(1-2 \epsilon, 2 \epsilon+1 ; 2(\epsilon+1) ;-1)\right.\right. \\
& -2 \epsilon-1))+6 \epsilon(1-2 \epsilon)\left(16^{\epsilon}{ }_{3} F_{2}(1,1-4 \epsilon, 2-2 \epsilon ; 2-4 \epsilon, 2 \epsilon+2 ; 1 / 2)\right. \\
& \left.\left.-2{ }_{3} F_{2}(1,1-4 \epsilon, 2-2 \epsilon ; 2-4 \epsilon, 2 \epsilon+2 ; 1)\right)\right]
\end{aligned}
$$

The diagram $L_{1}^{3}$ has to be computed along the contour (49), then the integral (46) becomes

$$
\begin{aligned}
L_{1}^{3} & =g(\epsilon)^{3} M^{2} N^{2} \cos \frac{\theta}{2} \frac{(1-2 \epsilon)^{3}}{2^{6 \epsilon}} \int_{0}^{1} d \tau_{1>2>3>4>5} \int_{0}^{1} d \tau_{6} \frac{1}{\left[\left(\tau_{3}-\tau_{4}\right)^{2}\left(\tau_{2}-\tau_{5}\right)^{2}\left(\tau_{1}+\tau_{6}\right)^{2}\right]^{1-2 \epsilon}} \\
& =\frac{g(\epsilon)^{3} M^{2} N^{2} \cos \frac{\theta}{2}}{3 \times 2^{4+6 \epsilon}} \frac{1-2 \epsilon}{\epsilon^{3}(1-4 \epsilon)}\left[1-3 \times 4^{\epsilon}-3{ }_{2} F_{1}(-2 \epsilon, 4 \epsilon ; 1+4 \epsilon ;-1)\right]
\end{aligned}
$$

The diagram $L_{1}^{4}$ has to be computed along the contour (50), then the integral (46) becomes

$$
\begin{aligned}
L_{1}^{4} & =g(\epsilon)^{3} M^{2} N^{2} \frac{(1-2 \epsilon)^{3}}{2^{6 \epsilon}} \int_{0}^{1} d \tau_{1>2>3>4>5>6} \frac{1}{\left[\left(\tau_{3}-\tau_{4}\right)^{2}\left(\tau_{2}-\tau_{5}\right)^{2}\left(\tau_{1}-\tau_{6}\right)^{2}\right]^{1-2 \epsilon}} \\
& =-\frac{g(\epsilon)^{3} M^{2} N^{2}}{3 \times 2^{4+6 \epsilon}} \frac{(1-2 \epsilon)^{2}}{\epsilon^{3}(1-4 \epsilon)(1-6 \epsilon)}
\end{aligned}
$$

Summing up the results using the formula (51) and expanding the result around $\epsilon=0$, the diagram $L_{1}^{\uparrow}$ is given by

$$
\begin{aligned}
& L_{1}^{\uparrow}=g(\epsilon)^{3} M^{2} N^{2}\left[\frac{c_{\theta}\left(\left(c_{\theta}-2\right) c_{\theta}+2\right)-2}{48 \epsilon^{3}}+\frac{c_{\theta}\left(c_{\theta}\left(-3 c_{\theta}(l+1)+6 l+4\right)-6 l+2\right)-6}{24 \epsilon^{2}}\right. \\
& +\frac{c_{\theta}^{3}\left(3 l(l+6)+2\left(\pi^{2}-2\right)\right)-2 c_{\theta}^{2}\left(3 l(l+4)+2\left(\pi^{2}-4\right)\right)+2 c_{\theta}\left(-3 l(l+2)+\pi^{2}+4\right)-40}{24 \epsilon} \\
& +\frac{1}{12}\left(c_{\theta}^{3}\left(3 l\left(l^{2}+l+4\right)-2 \pi^{2}(l+2)-39 \zeta(3)+16\right)+c_{\theta}^{2}\left(2 \pi^{2}(l+1)-2 l(l(5 l+12)+24)+72 \zeta(3)+32\right)\right. \\
& \left.\left.+c_{\theta}\left(-2 l(l(l+3)+12)-33 \zeta(3)+2 \pi^{2}+16\right)-128\right)+\mathcal{O}(\epsilon)\right]
\end{aligned}
$$

where we have introduced the short-hand notation $l=\log 2$ and $c_{\theta}=\cos \frac{\theta}{2}$.

The remaining fermionic diagrams can be computed in the same way. The closed expression and the $\epsilon$-expansion of any diagram can be found in the attached file "Ladders.nb". The complete fermionic ladder contribution to the expectation value of the cusped Wilson line can be computed with the following formula

$$
L_{F}^{\uparrow}=L_{1}^{\uparrow}+L_{2}^{\uparrow}+2 L_{3}^{\uparrow}+L_{5}^{\uparrow}
$$


and it reads

$$
\begin{aligned}
& L_{F}^{\uparrow}=g(\epsilon)^{3} M N\left[\frac{6\left(3 c_{\theta}-4\right) M^{2}+\left(c_{\theta}\left(\left(c_{\theta}-8\right) c_{\theta}+20\right)-20\right) M N-2\left(c_{\theta}\left(2 c_{\theta}-3\right)+2\right) N^{2}}{48 \epsilon^{3}}\right. \\
& +\frac{-3 c_{\theta}^{3}(l+1) M N+2 c_{\theta}^{2} N(9 l M+6 l N+5 M+2 N)-2 c_{\theta}\left(9 l(M+N)^{2}-4 M N\right)-8 N(3 M+N)}{24 \epsilon^{2}} \\
& +\frac{1}{72 \epsilon}\left(-2 N^{2}\left(9\left(4 c_{\theta}^{2}(l-1)+3 c_{\theta} l^{2}+8\right)+\pi^{2}\left(c_{\theta}\left(7 c_{\theta}-6\right)-2\right)\right)+3 M N\left(c_{\theta}^{3}\left(3 l(l+6)+2\left(\pi^{2}-2\right)\right)\right.\right. \\
& \left.\left.+c_{\theta}^{2}\left(6(l-8) l-6 \pi^{2}+40\right)-8 c_{\theta}\left(9 l^{2}+3 l-4\right)+8\left(\pi^{2}-14\right)\right)-6 M^{2}\left(27 c_{\theta} l^{2}+\pi^{2}\left(5 c_{\theta}-8\right)\right)\right) \\
& +\frac{1}{36}\left(3 c_{\theta}^{3} M N\left(3\left(l^{2}+l+4\right) l-2 \pi^{2} l-39 \zeta(3)-4 \pi^{2}+16\right)-c_{\theta}^{2} N\left(3 M \left(2 l\left(7 l^{2}+30 l+3 \pi^{2}+48\right)-75 \zeta(3)\right.\right.\right. \\
& \left.-80)+2 N\left(3 l\left(6 l(l+3)+\pi^{2}+36\right)+2\left(-51 \zeta(3)+\pi^{2}-54\right)\right)\right)+6 c_{\theta}\left(l^{3}\left(-\left(27 M^{2}+26 M N+3 N^{2}\right)\right)\right. \\
& \left.-12 l^{2} M N+l\left(\pi^{2}\left(9 M^{2}+10 M N+2 N^{2}\right)-24 M N\right)+32 M N+6 \zeta(3)\left(12 M^{2}+3 M N-5 N^{2}\right)\right) \\
& \left.\left.+8 N\left(3\left(\pi^{2}-34\right) M+\left(\pi^{2}-54\right) N\right)-48 \zeta(3)\left(15 M^{2}+9 M N+N^{2}\right)\right)+\mathcal{O}(\epsilon)\right]
\end{aligned}
$$

\section{Bosonic ladder diagrams}

The second class of diagrams can be computed performing the planar Wick contractions of the mixed bosonic-fermionic monomial (42). In order to do it using the package WiLE we have to set the initial data to

$$
\left(n_{f}=2, n_{g}=0, n_{s}=2\right) \quad \text { and } \quad V_{i}=0 \quad \text { with } \quad i=1,2, \ldots, 7 .
$$

As the previoue case, we set the options Supermatrix Sector and Planar on UL and on respectively and the remaining options on off. Given all the initial data the WiLE output is the following

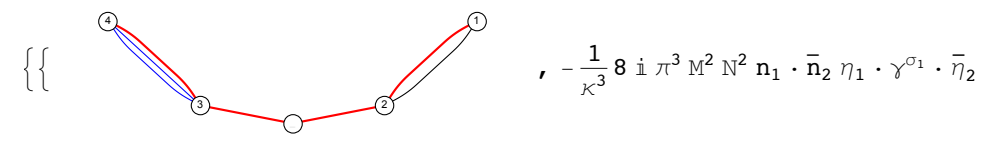

$$
\left.\mathrm{d}_{\mathbf{x}_{2}}{ }^{\sigma_{1}}\left[\Delta\left[\mathbf{x}_{2}, \mathbf{x}_{1}\right]\right] \bmod \left[\dot{\mathbf{x}}_{1}\right] \bmod \left[\dot{\mathbf{x}}_{2}\right] \bmod \left[\dot{\mathbf{x}}_{3}\right] \bmod \left[\dot{\mathbf{x}}_{4}\right] \operatorname{tr}\left[\mathrm{M}_{3} \cdot \mathrm{M}_{4}\right] \Delta\left[\mathbf{x}_{3}, \mathbf{x}_{4}\right] \Delta\left[\mathbf{x}_{4}, \mathbf{x}_{3}\right]\right\},
$$

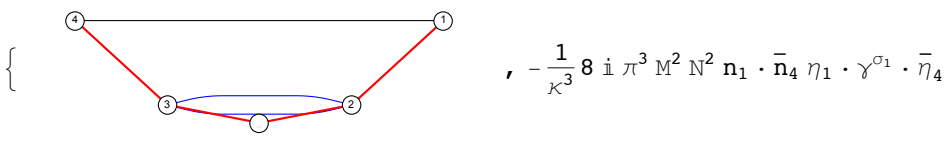

$\left.\mathrm{d}_{\mathrm{x}_{4}}{ }^{\sigma_{1}}\left[\Delta\left[\mathrm{x}_{4}, \mathrm{x}_{1}\right]\right] \bmod \left[\dot{\mathbf{x}}_{1}\right] \bmod \left[\dot{\mathbf{x}}_{2}\right] \bmod \left[\dot{\mathbf{x}}_{3}\right] \bmod \left[\dot{\mathbf{x}}_{4}\right] \operatorname{tr}\left[\hat{\mathrm{M}}_{2} \cdot \hat{\mathrm{M}}_{3}\right] \Delta\left[\mathrm{x}_{2}, \mathrm{x}_{3}\right] \Delta\left[\mathrm{x}_{3}, \mathrm{x}_{2}\right]\right\}$,

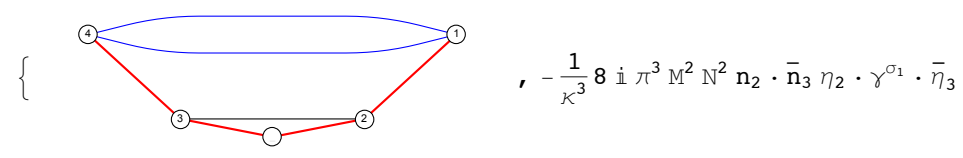

$\left.\mathrm{d}_{\mathrm{x}_{3}}{ }^{\sigma_{1}}\left[\Delta\left[\mathrm{x}_{3}, \mathrm{x}_{2}\right]\right] \bmod \left[\dot{\mathbf{x}}_{1}\right] \bmod \left[\dot{\mathbf{x}}_{2}\right] \bmod \left[\dot{\mathbf{x}}_{3}\right] \bmod \left[\dot{\mathbf{x}}_{4}\right] \operatorname{tr}\left[\mathrm{M}_{1} \cdot \mathrm{M}_{4}\right] \Delta\left[\mathrm{x}_{1}, \mathrm{x}_{4}\right] \Delta\left[\mathrm{x}_{4}, \mathrm{x}_{1}\right]\right\}$,

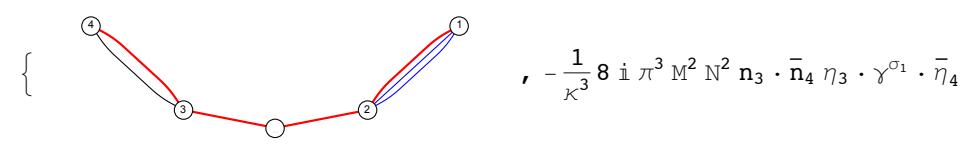

$$
\left.\left.\mathrm{d}_{\mathrm{x}_{4}}{ }^{\sigma_{1}}\left[\Delta\left[\mathrm{x}_{4}, \mathrm{x}_{3}\right]\right] \bmod \left[\dot{\mathrm{x}}_{1}\right] \bmod \left[\dot{\mathrm{x}}_{2}\right] \bmod \left[\dot{\mathrm{x}}_{3}\right] \bmod \left[\dot{\mathrm{x}}_{4}\right] \operatorname{tr}\left[\mathrm{M}_{1} \cdot \mathrm{M}_{2}\right] \Delta\left[\mathrm{x}_{1}, \mathrm{x}_{2}\right] \Delta\left[\mathrm{x}_{2}, \mathrm{x}_{1}\right]\right\}\right\}
$$

In order to simplify the following discussion we name the diagrams above $L_{i}^{\uparrow} i=6, \ldots, 9$ where the index $i$ corresponds to the raw of the WiLE output. For this class of diagrams, we have to 


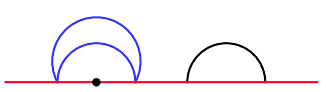

(a) $L_{6}^{1}$

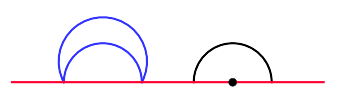

(b) $L_{6}^{2}$

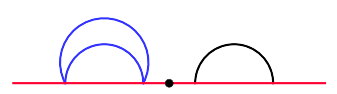

(c) $L_{6}^{3}$

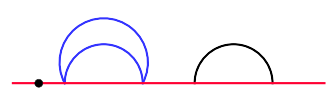

(d) $L_{6}^{4}$

Figure 3: Bosonic ladder diagrams contributing to the diagram $L_{6}$.

split the integration on the Wilson line in five subregions. Using the reflection property of the diagrams we have

$$
L_{9}^{\uparrow}=L_{6}^{\uparrow} .
$$

We will compute in detail the diagram $L_{6}$ as an example.

Following the prescriptions of section 3 and the legend of appendix D, the diagram $L_{6}$ is given by

$$
L_{6}^{\uparrow}=-i\left(\frac{2 \pi}{\kappa}\right)^{3} M^{2} N^{2} \int_{-L}^{L} d \tau_{1>\ldots>4}\left[\left|\dot{x}_{1}\left\|\dot{x}_{2}\right\| \dot{x}_{3} \| \dot{x}_{4}\right| \operatorname{tr}\left(\mathcal{M}_{3} \mathcal{M}_{4}\right)\left(n_{1} \bar{n}_{2}\right)\left(\eta_{1} \gamma^{\mu} \bar{\eta}_{2}\right) \partial_{x_{2}}^{\mu} \Delta\left(x_{2}, x_{1}\right) \Delta^{2}\left(x_{3}, x_{4}\right)\right]
$$

Because of the reflection property of the diagrams, we have to compute the integral (62) only for the integration regions depicted in Figure 3, namely

$$
\begin{array}{ll}
L_{6}^{1} & \left(-L<\tau_{4}<0\right) \in C_{1} \cup\left(0<\tau_{3}<\tau_{2}<\tau_{1}<L\right) \in C_{2} \\
L_{6}^{2} & \left(-L<\tau_{4}<\tau_{3}<\tau_{2}<0\right) \in C_{1} \cup\left(0<\tau_{1}<L\right) \in C_{2} \\
L_{6}^{3} & \left(-L<\tau_{4}<\tau_{3}<0\right) \in C_{1} \cup\left(0<\tau_{2}<\tau_{1}<L\right) \in C_{2} \\
L_{6}^{4} & \left(0<\tau_{4}<\tau_{3}<\tau_{2}<\tau_{1}<L\right) \in C_{2}
\end{array}
$$

then the total result can be computed using the following formula

$$
L_{6}^{\uparrow}=L_{6}^{1}+L_{6}^{2}+L_{6}^{3}+2 L_{6}^{4} .
$$

Following the same steps of the fermionic case, the integral (62) along the contour (63) is given by

$$
\begin{aligned}
L_{6}^{1} & =g(\epsilon)^{3} M^{2} N^{2} \cos ^{2} \frac{\theta}{2} \frac{(1-2 \epsilon)}{2^{6 \epsilon}} \int_{0}^{1} d \tau_{1>2>3} \int_{0}^{1} d \tau_{4} \frac{1}{\left[\left(\tau_{1}-\tau_{2}\right)^{2}\right]^{1-\epsilon}} \frac{1}{\left[\left(\tau_{3}+\tau_{4}\right)^{2}\right]^{1-2 \epsilon}} \\
& \left.=-\frac{g(\epsilon)^{3} M^{2} N^{2} \cos ^{2} \frac{\theta}{2}}{2^{3+6 \epsilon} \epsilon^{2}(1+2 \epsilon)(1-4 \epsilon)}\left[1+\frac{\Gamma(1+2 \epsilon) \Gamma(1+4 \epsilon)}{\Gamma(1+6 \epsilon)}-{ }_{2} F_{1}(1,-4 \epsilon ; 1+2 \epsilon ;-1)\right)\right]
\end{aligned}
$$

The diagram $L_{6}^{2}$ has to be computed along the contour (64) and it reads

$$
\begin{aligned}
L_{6}^{2} & =g(\epsilon)^{3} M^{2} N^{2} \cos \frac{\theta}{2} \frac{(1-2 \epsilon)}{2^{6 \epsilon}} \int_{0}^{1} d \tau_{1} \int_{0}^{1} d \tau_{4>3>2} \frac{1}{\left[\left(\tau_{1}+\tau_{2}\right)^{2}\right]^{1-\epsilon}} \frac{1}{\left[\left(\tau_{4}-\tau_{3}\right)^{2}\right]^{1-2 \epsilon}} \\
& \left.=-\frac{g(\epsilon)^{3} M^{2} N^{2} \cos \frac{\theta}{2}}{2^{3+6 \epsilon} \epsilon^{2}(1-4 \epsilon)}\left[1+\frac{\Gamma(1+2 \epsilon) \Gamma(1+4 \epsilon)}{\Gamma(1+6 \epsilon)}-{ }_{2} F_{1}(1,-2 \epsilon ; 1+4 \epsilon ;-1)\right)\right]
\end{aligned}
$$


The diagram $L_{6}^{3}$ has to be computed along the contour (65) and it reads

$$
\begin{aligned}
L_{6}^{3} & =g(\epsilon)^{3} M^{2} N^{2} \frac{(1-2 \epsilon)}{2^{6 \epsilon}} \int_{0}^{1} d \tau_{1>2} \int_{0}^{1} d \tau_{4>3} \frac{1}{\left[\left(\tau_{1}-\tau_{2}\right)^{2}\right]^{1-\epsilon}} \frac{1}{\left[\left(\tau_{4}-\tau_{3}\right)^{2}\right]^{1-2 \epsilon}} \\
& =\frac{g(\epsilon)^{3} M^{2} N^{2}}{2^{3+6 \epsilon} \epsilon^{2}(1-4 \epsilon)}
\end{aligned}
$$

Finally the diagram $L_{6}^{4}$ has to be computed along the contour (66) and it is given by

$$
\begin{aligned}
L_{6}^{4} & =g(\epsilon)^{3} M^{2} N^{2} \frac{(1-2 \epsilon)}{2^{6 \epsilon}} \int_{0}^{1} d \tau_{1>2>3>4} \frac{1}{\left[\left(\tau_{1}-\tau_{2}\right)^{2}\right]^{1-\epsilon}} \frac{1}{\left[\left(\tau_{3}-\tau_{4}\right)^{2}\right]^{1-2 \epsilon}} \\
& =-\frac{g(\epsilon)^{3} M^{2} N^{2}}{2^{6 \epsilon}} \frac{\Gamma(2 \epsilon) \Gamma(4 \epsilon-1)}{\Gamma(1+6 \epsilon)}
\end{aligned}
$$

Summing up the results using the formula (67) and expanding the result around $\epsilon=0$, the diagram $L_{6}^{\uparrow}$ is given by

$$
\begin{aligned}
L_{6}^{\uparrow}=g(\epsilon)^{3} & M^{2} N^{2}\left[\frac{3-c_{\theta}\left(c_{\theta}+1\right)}{8 \epsilon^{2}}+\frac{c_{\theta}\left(2 c_{\theta}(l-1)+l-2\right)+6}{4 \epsilon}\right. \\
+ & \left.\frac{1}{12}\left(\pi^{2}\left(c_{\theta}^{2}+c_{\theta}-4\right)+3 c_{\theta}\left(2 c_{\theta}(l+2)(3 l-2)+l(3 l+4)-8\right)+72\right)\right]
\end{aligned}
$$

The remaining bosonic diagrams can be computed in the same way. The closed expression and the $\epsilon$-expansion of any diagram can be found in the attached file "Ladders.nb". The complete bosonic ladder contribution to the expectation value of the cusped Wilson line can be computed with the following formula

$$
L_{B}^{\uparrow}=2 L_{6}^{\uparrow}+L_{7}^{\uparrow}+L_{8}^{\uparrow}
$$

and it reads

$$
\begin{aligned}
L_{B}^{\uparrow}= & g(\epsilon)^{3} M^{2} N^{2}\left[\frac{\left(c_{\theta}-4\right) c_{\theta}\left(3 c_{\theta}+2\right)+24}{24 \epsilon^{2}}+\frac{3 c_{\theta}\left(c_{\theta}\left(8-3 c_{\theta}\right)+4\right) l+2\left(c_{\theta}\left(\left(c_{\theta}-10\right) c_{\theta}-8\right)+28\right)}{12 \epsilon}\right. \\
& \left.-\frac{1}{12}\left(c_{\theta}^{3}\left(3 l(l+4)-4\left(\pi^{2}-4\right)\right)-c_{\theta}^{2}(12 l(5 l+8)-80)-8 c_{\theta}(3 l(l+2)-8)+8\left(\pi^{2}-34\right)\right)\right]
\end{aligned}
$$

Summing up fermionic and bosonic ladders The total contribution of the ladder diagrams to the three-loop expectation value of the upper-left block of the Wilson loop can be computed using the following formula

$$
\left\langle\mathcal{W}^{\uparrow}[C]\right\rangle_{\text {ladder }}^{(3)}=L_{F}^{\uparrow}+L_{B}^{\uparrow}
$$


Recalling the prescription give in (14) and the relation (18), we are finally able to compute the expectation value of the fermionic operators $\mathcal{W}_{ \pm}$. The traced operator $\mathcal{W}_{+}$is given by

$$
\begin{aligned}
& \left\langle\mathcal{W}_{+}[C]\right\rangle_{\text {ladder }}^{(3)}=g(\epsilon)^{3} \frac{M N}{N+M}\left[\frac{-2\left(\left(c_{\theta}-6\right) c_{\theta}+7\right)\left(M^{2}+N^{2}\right)+\left(c_{\theta}\left(\left(c_{\theta}-8\right) c_{\theta}+20\right)-20\right) M N}{24 \epsilon^{3}}\right. \\
& +\frac{2\left(M^{2}+N^{2}\right)\left(c_{\theta}^{2}+3\left(c_{\theta}-3\right) c_{\theta} l-2\right)-3 c_{\theta}\left(\left(c_{\theta}-6\right) c_{\theta}+12\right) l M N}{12 \epsilon^{2}}-\frac{1}{36 \epsilon}\left(( M ^ { 2 } + N ^ { 2 } ) \left(3 6 \left(c_{\theta}^{2}(l-1)\right.\right.\right. \\
& \left.\left.\left.+3 c_{\theta} l^{2}+2\right)+\pi^{2}\left(c_{\theta}\left(7 c_{\theta}+9\right)-26\right)\right)-3 M N\left(3\left(c_{\theta}-4\right) c_{\theta}\left(c_{\theta}+6\right) l^{2}+2 \pi^{2}\left(c_{\theta}+1\right)\left(c_{\theta}-2\right)^{2}\right)\right) \\
& -\frac{1}{18}\left(( M ^ { 2 } + N ^ { 2 } ) \left(18 c_{\theta}\left(c_{\theta}(l(l(l+3)+6)-6)+5 l^{3}\right)-\pi^{2} c_{\theta}\left(33 l-c_{\theta}(3 l+2)\right)\right.\right. \\
& \left.-6\left(c_{\theta}\left(17 c_{\theta}+21\right)-64\right) \zeta(3)-4 \pi^{2}+216\right)+3 M N\left(c _ { \theta } l \left(\left(c_{\theta}\left(14-3 c_{\theta}\right)+52\right) l^{2}\right.\right. \\
& \left.\left.\left.\left.+2 \pi^{2}\left(c_{\theta}-2\right)\left(c_{\theta}+5\right)\right)+3\left(c_{\theta}\left(c_{\theta}\left(13 c_{\theta}-25\right)-12\right)+48\right) \zeta(3)\right)\right)+\mathcal{O}(\epsilon)\right],
\end{aligned}
$$

and the supertraced operator $\mathcal{W}_{-}$is given by

$$
\begin{aligned}
& \left\langle\mathcal{W}_{-}[C]\right\rangle_{\text {ladder }}^{(3)}=g(\epsilon)^{3} M N(M+N)\left[-\frac{\left(c_{\theta}\left(c_{\theta}+3\right)-5\right)}{12 \epsilon^{3}}+\frac{\left(c_{\theta}^{2}(3 l+1)-2\right)}{6 \epsilon^{2}}\right. \\
& -\frac{\left(18\left(2 c_{\theta}^{2}(l-1)-3 c_{\theta} l^{2}+4\right)+\pi^{2}\left(7\left(c_{\theta}-3\right) c_{\theta}+22\right)\right)}{36 \epsilon}-\frac{1}{18}\left(18 c_{\theta}\left(c_{\theta}(l(l(l+3)+6)-6)-4 l^{3}\right)\right. \\
& \left.\left.+\pi^{2} c_{\theta}\left(3\left(c_{\theta}+7\right) l+2 c_{\theta}\right)-\left(102\left(c_{\theta}-3\right) c_{\theta}+336\right) \zeta(3)-4 \pi^{2}+216\right)+\mathcal{O}(\epsilon)\right]
\end{aligned}
$$

The divergent part of (76), in the $M=N$ case, is in agreement with the same computation done in ABJM using the heavy quark effective theory formalism (HQET) in [41].

Recently, the ladder contribution to the expectation value of the cusped Wilson loops $\mathcal{W}_{ \pm}$was computed in [39] using the Bethe-Salpeter equation in the following scaling limit

$$
i \theta>>0, \quad \lambda_{1,2}<<1 \quad \text { with } \hat{\lambda}_{1,2}=\lambda_{1,2} \cos \frac{\theta}{2} \quad \text { fixed },
$$

where $\lambda_{1,2}$ are the 't Hooft coupling defined in (23). In this limit only the leading divergence of the ladders contributes and the perturbative series can be reorganized in powers of $\hat{\lambda}$. This allows to compute exactly $\mathcal{W}_{ \pm}$for the cusped contour. In the $\varphi=0$ case the solutions are the following

$$
\begin{aligned}
& \left\langle\mathcal{W}_{+}[C]\right\rangle=\frac{(\sqrt{M}+\sqrt{N})^{2}}{2(N+M)} e^{\frac{1}{2 \epsilon} \frac{\sqrt{N M c_{\theta}}}{\kappa}}+\frac{(\sqrt{M}-\sqrt{N})^{2}}{2(N+M)} e^{-\frac{1}{2 \epsilon} \frac{\sqrt{N M} c_{\theta}}{\kappa}}, \\
& \left\langle\mathcal{W}_{-}[C]\right\rangle=\frac{1}{2} e^{\frac{1}{2 \epsilon} \frac{\sqrt{N M} c_{\theta}}{\kappa}}+\frac{1}{2} e^{-\frac{1}{2 \epsilon} \frac{\sqrt{N M} c_{\theta}}{\kappa}} .
\end{aligned}
$$

Considering the scaling limit (78), the leading divergences of (76) and (77) are

$$
\left\langle\mathcal{W}_{+}[C]\right\rangle_{\text {ladder }}^{(3)} \stackrel{\text { s.l. }}{=} \frac{M^{2} N^{2} c_{\theta}^{3}}{24(M+N) \epsilon^{3} \kappa^{3}} \quad \text { and } \quad\left\langle\mathcal{W}_{-}[C]\right\rangle_{\text {ladder }}^{(3)} \stackrel{\text { s.l. }}{=} 0,
$$

that are in agreement with the three-loop expansion of (79). 


\section{Acknowledgments}

It is a pleasure to thank Lorenzo Bianchi, Luca Griguolo, Marco Meineri, Domenico Seminara and Edoardo Vescovi for very useful discussions and the critical reading of the draft. The work of MP is supported by "Della Riccia Foundation" grant.

\section{A Notation and conventions}

We work in Euclidean space in three dimensions with coordinates $x^{\mu}=\left\{x^{1}, x^{2}, x^{3}\right\}$. We choose a set of Dirac gamma matrices satisfying the three dimensional Clifford algebra as

$$
\left(\gamma^{\mu}\right)_{\alpha}^{\beta}=\left\{-\sigma^{3}, \sigma^{1}, \sigma^{2}\right\}
$$

where $\sigma^{i}$ are the Pauli matrices. In our notation, the product of matrices is given by

$$
\left(\gamma^{\mu} \gamma^{\nu}\right)_{\alpha}^{\beta} \equiv\left(\gamma^{\mu}\right)_{\alpha}^{\gamma}\left(\gamma^{\nu}\right)_{\gamma}^{\beta}
$$

The gamma matrices (81) satisfy the following relations

$$
\begin{aligned}
\gamma^{\mu} \gamma^{\nu} & =\delta^{\mu \nu} \mathbb{1}-i \epsilon^{\mu \nu \rho} \gamma^{\rho} & \operatorname{tr}\left(\gamma^{\mu} \gamma^{\nu}\right) & =2 \delta^{\mu \nu} \\
\gamma^{\mu} \gamma^{\nu} \gamma^{\rho} & =\delta^{\mu \nu} \gamma^{\rho}-\delta^{\mu \rho} \gamma^{\nu}+\delta^{\nu \rho} \gamma^{\mu}-i \epsilon^{\mu \nu \rho} \mathbb{1} & \operatorname{tr}\left(\gamma^{\mu} \gamma^{\nu} \gamma^{\rho}\right) & =-2 i \epsilon^{\mu \nu \rho}
\end{aligned}
$$

Spinor indices are lowered and raised with the usual $\epsilon$-tensor

$$
\psi^{\alpha}=\epsilon^{\alpha \beta} \psi_{\beta} \quad, \quad \psi_{\alpha}=\epsilon_{\alpha \beta} \psi^{\beta}
$$

where $\epsilon^{12}=-\epsilon_{12}=1$. Moreover, under complex conjugation the Dirac matrices transform as follows:

$$
\left[\left(\gamma^{\mu}\right)_{\alpha}^{\beta}\right]^{*}=\left(\gamma^{\mu}\right)_{\alpha}^{\beta} \equiv \epsilon^{\beta \gamma}\left(\gamma^{\mu}\right)_{\gamma}^{\delta} \epsilon_{\alpha \delta}
$$

During the evaluation of fermionic contributions to the Wilson loop vev, we often encounter bilinears constructed with the spinors $\eta$ and $\bar{\eta}$ defined by the two relations in (8). The simplest bilinear contains one Dirac gamma matrix and it can be expressed as a function of the position as follows

$$
\left(\eta_{2} \gamma^{\mu} \bar{\eta}_{1}\right)=-\frac{2}{\left(\eta_{1} \bar{\eta}_{2}\right)}\left[\frac{\dot{x_{1}}{ }^{\mu}}{\left|\dot{x}_{1}\right|}+\frac{\dot{x_{2}}{ }^{\mu}}{\left|\dot{x}_{2}\right|}-i \frac{\dot{x_{2}}{ }^{\lambda}}{\left|\dot{x}_{2}\right|} \frac{\dot{x}_{1}^{\nu}}{\left|\dot{x}_{1}\right|} \epsilon_{\lambda \nu}{ }^{\mu}\right]
$$

where the superscripts 1 and 2 denote two different points on the contour. Bilinears with a higher number of contracted gamma matrices can be rewritten in terms of the bilinear (86) and the spinors contraction $\eta \bar{\eta}$ using the relations (83) and their generalization.

The $U(N)$ gauge group generators $T^{A}=\left(T^{0}, T^{a}\right)$, where $T^{0}=\frac{\mathbb{1}}{\sqrt{N}}$ and $a=1, \ldots, N^{2}-1$, are an 
orthonormal set of traceless $N \times N$ hermitian matrices with the following normalization

$$
\operatorname{Tr}_{\mathbf{N}}\left(T^{A} T^{B}\right)=\delta^{A B}
$$

similarly for the set of $M \times M$ generators of $U(M)$. The WiLE package uses the double line notation in which the fields carry two indices in the fundamental representation of the two gauge groups. In general the lowercase roman indices $i, j, \ldots$ are in the fundamental representation of $U(N)$ and the hatted lowercase roman indices $\hat{i}, \hat{j}, \ldots$ are in the fundamental representation of $U(M)$.

\section{B The ABJM theory action}

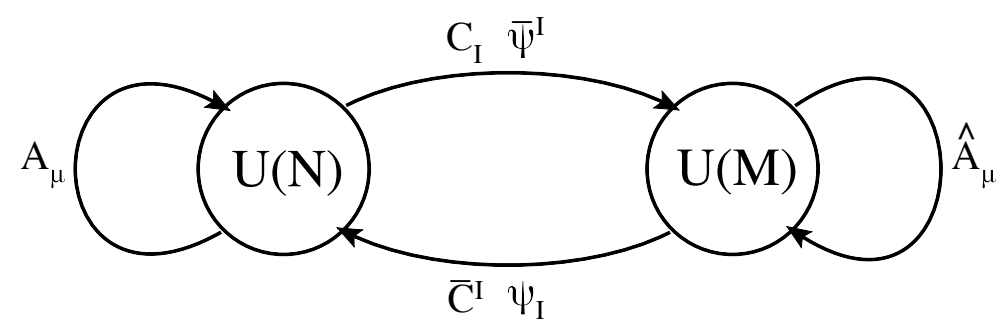

Figure 4: Quiver diagram for ABJ(M) theory.

The $\mathcal{N}=6$ super Chern-Simons-matter theory, also known as $\mathrm{ABJ}(\mathrm{M})$ in three dimensions, is a superconformal field theory with gauge group $U_{\kappa}(N) \times U_{-\kappa}(M)$ and global symmetry given by the orthosymplectic supergroup $\operatorname{OSp}(6 \mid 4)$ [26, 54]. The bosonic part of the supergroup involves the R-symmetry group $S O(6) \sim S U(4)$ and the three-dimensional conformal group $S p(4) \sim S O(2,3)$. The fermionic part generates the $\mathcal{N}=6$ supersymmetries.

The field content of the $\mathrm{ABJ}(\mathrm{M})$ theory can be schematically represented by the quiver in Figure 4. The gauge fields $\left(A_{\mu}\right)_{i}{ }^{j}$ and $\left(\hat{A}_{\mu}\right)_{\hat{i}}^{\hat{j}}$, belonging respectively to the adjoint of $U_{\kappa}(N)$ and $U_{-\kappa}(M)$, are represented by arrows forming a loop with their gauge group sector. The matter sector instead contains a couple of complex scalars $\left(C_{I}\right)_{i}{ }^{\hat{j}}$ and $\left(\bar{C}^{I}\right)_{\hat{i}}^{j}$ as well as the fermions $\left(\psi_{I}\right)_{\hat{i}}^{j}$ and $\left(\bar{\psi}^{I}\right)_{i}{ }^{\hat{j}}$. The matter fields are represented by arrows connecting the two different side of the gauge group $U_{\kappa}(N) \times U_{-\kappa}(M)$, indeed the fields $(C, \bar{\psi})$ transform in the $(\mathbf{N}, \overline{\mathbf{M}})$ representation while the pair $(\bar{C}, \psi)$ in the $(\overline{\mathbf{N}}, \mathbf{M})$. The additional capital index $I=1, \ldots, 4$ belongs to the R-symmetry group $S U(4)$. We have also to introduce the covariant gauge fixing function $\partial_{\mu} A^{\mu}$ for both gauge fields and two sets of ghosts $(\bar{c}, c)$ and $(\overline{\hat{c}}, \hat{c})$, in order to quantize the theory at the perturbative level. Then the ABJ(M) action can be written as the sum of the following terms

$$
S_{\mathrm{ABJ}(\mathrm{M})}=S_{\mathrm{CS}}+S_{\mathrm{gf}}+S_{\mathrm{matter}}+S_{\mathrm{pot}}^{F}+S_{\mathrm{pot}}^{B}
$$

namely the Chern-Simons action, the gauge-fixing action, the matter term, a fermionic and a 
bosonic potential. We work with the following Euclidian space action

$$
\begin{aligned}
S_{\mathrm{CS}} & =-i \frac{\kappa}{4 \pi} \int d^{3} x \epsilon^{\mu \nu \rho}\left[\operatorname{Tr}\left(A_{\mu} \partial_{\nu} A_{\rho}+\frac{2}{3} i A_{\mu} A_{\nu} A_{\rho}\right)-\operatorname{Tr}\left(\hat{A}_{\mu} \partial_{\nu} \hat{A}_{\rho}+\frac{2}{3} i \hat{A}_{\mu} \hat{A}_{\nu} \hat{A}_{\rho}\right)\right], \\
S_{\text {gf }} & =\frac{\kappa}{4 \pi} \int d^{3} x\left[\frac{1}{\xi} \operatorname{Tr}\left(\partial_{\mu} A^{\mu}\right)^{2}+\operatorname{Tr}\left(\partial_{\mu} \bar{c} D^{\mu} c\right)-\frac{1}{\xi} \operatorname{Tr}\left(\partial_{\mu} \hat{A}^{\mu}\right)^{2}-\operatorname{Tr}\left(\partial_{\mu} \overline{\hat{c}} D^{\mu} \hat{c}\right)\right], \\
S_{\text {matter }} & =\int d^{3} x\left[\operatorname{Tr}\left(D_{\mu} C_{I} D^{\mu} \bar{C}^{I}\right)+i \operatorname{Tr}\left(\bar{\psi}^{I} \not D \psi_{I}\right)\right],
\end{aligned}
$$

and the following potentials

$$
\begin{gathered}
S_{\text {pot }}^{F}=-\frac{2 \pi i}{\kappa} \int d^{3} x\left[\operatorname{Tr}\left(\bar{C}^{I} C_{I} \psi_{J} \bar{\psi}^{J}\right)-\operatorname{Tr}\left(C_{I} \bar{C}^{I} \bar{\psi}^{J} \psi_{J}\right)+2 \operatorname{Tr}\left(C_{I} \bar{C}^{J} \bar{\psi}^{I} \psi_{J}\right)\right. \\
\left.-2 \operatorname{Tr}\left(\bar{C}^{I} C_{J} \psi_{I} \bar{\psi}^{J}\right)-\epsilon_{I J K L} \operatorname{Tr}\left(\bar{C}^{I} \bar{\psi}^{J} \bar{C}^{K} \bar{\psi}^{L}\right)+\epsilon^{I J K L} \operatorname{Tr}\left(C_{I} \psi_{J} C_{K} \psi_{L}\right)\right], \\
S_{\text {pot }}^{B}=-\frac{4 \pi^{2}}{3 \kappa^{2}} \int d^{3} x\left[\operatorname{Tr}\left(C_{I} \bar{C}^{I} C_{J} \bar{C}^{J} C_{K} \bar{C}^{K}\right)+\operatorname{Tr}\left(\bar{C}^{I} C_{I} \bar{C}^{J} C_{J} \bar{C}^{K} C_{K}\right)\right. \\
\left.+4 \operatorname{Tr}\left(C_{I} \bar{C}^{J} C_{K} \bar{C}^{I} C_{J} \bar{C}^{K}\right)-6 \operatorname{Tr}\left(C_{I} \bar{C}^{J} C_{J} \bar{C}^{I} C_{K} \bar{C}^{K}\right)\right],
\end{gathered}
$$

where $\epsilon^{1234}=\epsilon_{1234}=1$ and $\kappa$ is the integer Chern-Simons level. The matter covariant derivatives are defined as

$$
\begin{aligned}
D_{\mu} C_{I}=\partial_{\mu} C_{I}+i\left(A_{\mu} C_{I}-C_{I} \hat{A}_{\mu}\right), & D_{\mu} \bar{C}^{I}=\partial_{\mu} \bar{C}^{I}-i\left(\bar{C}^{I} A_{\mu}-\hat{A}_{\mu} \bar{C}^{I}\right), \\
D_{\mu} \psi_{I}=\partial_{\mu} \psi_{I}+i\left(\hat{A}_{\mu} \psi_{I}-\psi_{I} A_{\mu}\right), & D_{\mu} \bar{\psi}^{I}=\partial_{\mu} \bar{\psi}^{I}-i\left(\bar{\psi}^{I} \hat{A}_{\mu}-A_{\mu} \bar{\psi}^{I}\right) .
\end{aligned}
$$

The interaction vertices $V_{i}$ can be read directly from the action (88) as mentioned in the section 3.2 .

The action given by (88) is invariant under the following supersymmetry transformations

$$
\begin{aligned}
\delta A_{\mu}= & \frac{4 \pi i}{k} \bar{\theta}^{I J \alpha}\left(\gamma_{\mu}\right)_{\alpha}^{\beta}\left(C_{I} \psi_{J \beta}+\frac{1}{2} \epsilon_{I J K L} \bar{\psi}_{\beta}^{K} \bar{C}^{L}\right), \\
\delta \hat{A}_{\mu}= & \frac{4 \pi i}{k} \bar{\theta}^{I J \alpha}\left(\gamma_{\mu}\right)_{\alpha}^{\beta}\left(\psi_{J \beta} C_{I}+\frac{1}{2} \epsilon_{I J K L} \bar{C}^{L} \bar{\psi}_{\beta}^{K}\right), \\
\delta C_{K}= & \bar{\theta}^{I J \alpha} \epsilon_{I J K L} \bar{\psi}_{\alpha}^{L}, \\
\delta \bar{C}^{K}= & 2 \bar{\theta}^{K L \alpha} \psi_{L \alpha}, \\
\delta \psi_{K}^{\beta}= & -i \bar{\theta}^{I J \alpha} \epsilon_{I J K L}\left(\gamma^{\mu}\right)_{\alpha}^{\beta} D_{\mu} \bar{C}^{L} \\
& \quad+\frac{2 \pi i}{k} \bar{\theta}^{I J \beta} \epsilon_{I J K L}\left(\bar{C}^{L} C_{P} \bar{C}^{P}-\bar{C}^{P} C_{P} \bar{C}^{L}\right)+\frac{4 \pi i}{k} \bar{\theta}^{I J \beta} \epsilon_{I J M L} \bar{C}^{M} C_{K} \bar{C}^{L}, \\
\delta \bar{\psi}_{\beta}^{K}= & -2 i \bar{\theta}^{K L \alpha}\left(\gamma^{\mu}\right)_{\alpha \beta} D_{\mu} C_{L}-\frac{4 \pi i}{k} \bar{\theta}_{\beta}^{K L}\left(C_{L} \bar{C}^{M} C_{M}-C_{M} \bar{C}^{M} C_{L}\right)-\frac{8 \pi i}{k} \bar{\theta}_{\beta}^{I J} C_{I} \bar{C}^{K} C_{J},
\end{aligned}
$$

where the parameter $\theta$ was written in terms of $\bar{\theta}$ using

$$
\theta_{I J}=\frac{1}{2} \epsilon_{I J K L} \bar{\theta}^{K L}
$$

Both the supersymmetry parameters are antisymmetric in $I \leftrightarrow J$, and they satisfy the reality condition $\bar{\theta}^{I J}=\left(\theta_{I J}\right)^{*}$. 


\section{Feynman rules}

The WiLE output is written in terms of position-space propagators $\Delta$, which can be computed from those in momentum space [28] by means of the following relation

$$
\int \frac{d^{3-2 \epsilon} p}{(2 \pi)^{3-2 \epsilon}} \frac{e^{i p \cdot x}}{\left(p^{2}\right)^{s}}=\frac{\Gamma\left(\frac{3}{2}-s-\epsilon\right)}{4^{s} \pi^{\frac{3}{2}-\epsilon} \Gamma(s)} \frac{1}{\left(x^{2}\right)^{\frac{3}{2}-s-\epsilon}} .
$$

Defining the quantity

$$
\Delta(x, y) \equiv \frac{\Gamma\left(\frac{1}{2}-\epsilon\right)}{4 \pi^{\frac{3}{2}-\epsilon}} \frac{1}{\left((x-y)^{2}\right)^{\frac{1}{2}-\epsilon}},
$$

in Landau gauge, we have the following propagators

$$
\begin{aligned}
\left\langle\left(A_{\mu}\right)_{i}^{j}(x)\left(A_{\nu}\right)_{k}^{l}(y)\right\rangle & =-\left(\frac{2 \pi i}{\kappa}\right) \delta_{i}^{l} \delta_{k}^{j} \epsilon_{\mu \nu \rho} \partial_{x}^{\rho} \Delta(x, y), \\
\left\langle\left(\hat{A}_{\mu}\right)_{\hat{i}} \hat{j}^{j}(x)\left(\hat{A}_{\nu}\right)_{\hat{k}}^{\hat{l}}(y)\right\rangle & =\left(\frac{2 \pi i}{\kappa}\right) \delta_{\hat{i}}^{l} \delta_{\hat{k}}^{j} \epsilon_{\mu \nu \rho} \partial_{x}^{\rho} \Delta(x, y), \\
\left\langle\left(C_{I}\right)_{i}{ }^{\hat{j}}(x)\left(\bar{C}^{J}\right)_{\hat{k}}^{l}(y)\right\rangle & =\delta_{I}^{J} \delta_{i}^{l} \delta_{\hat{k}}^{\hat{j}} \Delta(x, y), \\
\left\langle\left(\psi_{I}\right)_{\hat{i}}^{j}(x)\left(\bar{\psi}^{J}\right)_{k}^{\hat{l}}(y)\right\rangle & =-i \delta_{I}^{J} \delta_{\hat{i}}^{\hat{l}} \delta_{k}^{j} \partial_{x} \Delta(x, y), \\
\left\langle(c)_{i}^{j}(x)(\bar{c})_{k}^{l}(y)\right\rangle & =-\left(\frac{4 \pi}{\kappa}\right) \delta_{i}^{l} \delta_{k}^{j} \Delta(x, y), \\
\left\langle(\hat{c})_{\hat{i}} \hat{j}(x)(\overline{\hat{c}})_{\hat{k}}^{\hat{l}}(y)\right\rangle & =\left(\frac{4 \pi}{\kappa}\right) \delta_{\hat{i}}^{\hat{l}} \delta_{\hat{k}}^{\hat{j}} \Delta(x, y) .
\end{aligned}
$$

\section{From WiLE output to physical variables}

In this section we present the complete list of the symbols appearing in the WiLE output and their physical meaning.

\section{Feynman diagrams}

1. Wilson loop contour

- $\longrightarrow \quad$ Wilson loop contour line,

$-\longrightarrow$ Additional vertex only for graphical purposes when the number of fields on the loop is even.

2. Fields and vertices positions

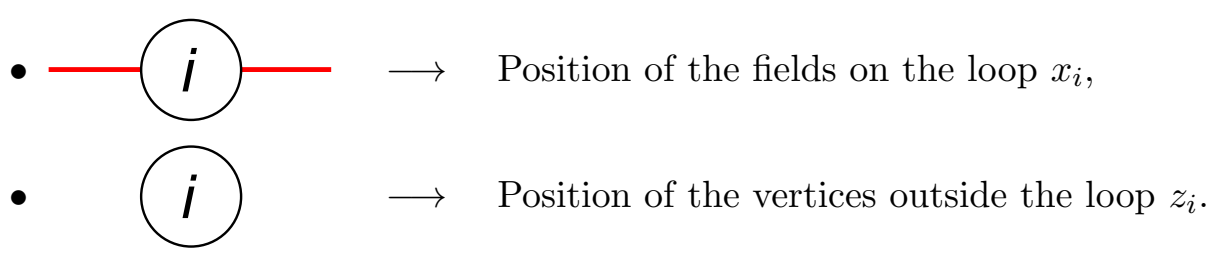


3. Propagators

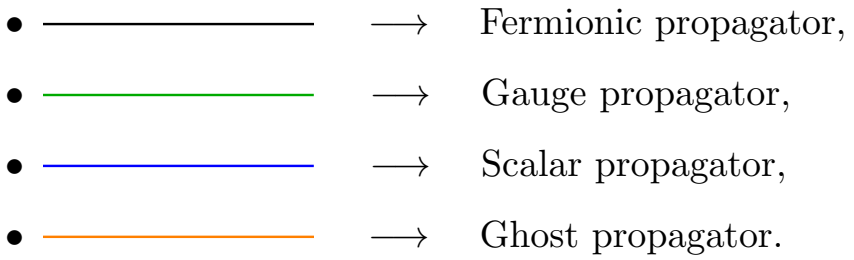

\section{Diagrams integral representation}

Each entry of the following legend is organized as follows

$$
\text { WiLE output form } \longrightarrow \text { Mathematica full form } \longrightarrow \text { Physical vairable }
$$

Notice that the package functions contain many greek characters $\backslash[$ name $]$. They can be typed into the Mathematica ${ }^{\circledR}$ notebook using the shortcut Esc+name+Esc.

1. The Chern-Simons level $\kappa$ and the gauge group ranks $N$ and $M$

- Out[1]= K $\longrightarrow \backslash$ [Kappa $] \quad \longrightarrow \kappa$,

- Out[1]= N $\longrightarrow \backslash$ [CapitalNu] $\longrightarrow N$,

- Out[1]= M $\longrightarrow \backslash$ [CapitalMu $] \longrightarrow M$.

2. Lorentz ${ }^{13}$ and R-symmetry indices $\mu_{i}$ and $J_{i}$

- Out[1]= $\mu_{i} \longrightarrow \backslash[\mathrm{Mu}][\mathrm{i}] \longrightarrow \mu_{i}$,

- Out[1]= $\mathrm{J}_{\mathrm{i}} \longrightarrow \mathrm{J}[\mathrm{i}] \quad \longrightarrow J_{i}$.

3. The position of the fields on the Wilson loop contour $x_{i}$, the derivative of the position respect to $\tau_{i} \dot{x}_{i}^{\mu}$, its module $\left|\dot{x}_{i}\right|$ and the vertices position $z_{i}$
- Out[1] $=\mathrm{x}_{\mathrm{i}}$
$\longrightarrow \quad \mathrm{x}[\backslash[\mathrm{Tau}][\mathrm{i}]]$
$\longrightarrow \quad x_{i}$,
- Out[1] $=\dot{\mathrm{x}}_{i}^{\mu}$
$\longrightarrow \quad \operatorname{xp}[\backslash[\mathrm{Tau}][\mathrm{i}], \backslash[\mathrm{Mu}]] \longrightarrow \dot{x}_{i}^{\mu}$,
- Out[1]= $\bmod \left[\dot{\mathrm{x}}_{i}\right.$
$\longrightarrow \bmod [\operatorname{xp}[\backslash[\mathrm{Tau}][\mathrm{i}]]] \quad \longrightarrow \quad\left|\dot{x}_{i}\right|$,
- Out[1]= $\mathbf{z}_{i}$
$\longrightarrow \quad z[i]$
$\longrightarrow z_{i}$

4. The Dirac gamma matrices $\gamma^{\mu}$

- Out[1]= $\gamma^{\mu} \longrightarrow \backslash[$ Gamma $][\backslash[\mathrm{Mu}]] \longrightarrow \gamma^{\mu}$.

5. The Levi-Civita tensors with Lorentz indices $\epsilon_{\mu \nu \rho}$ and with R-symmetry indices $\epsilon_{I J K L}$

- Out[1]= $\epsilon_{\mu \nu \rho} \longrightarrow \quad \backslash[$ Epsilon $][\backslash[\mathrm{Mu}], \backslash[\mathrm{Nu}], \backslash[\mathrm{Rho}]] \longrightarrow \epsilon_{\mu \nu \rho}$,

- Out[1] $=\epsilon_{\mathrm{IJKL}} \longrightarrow \backslash[$ Epsilon $] \backslash[$ Epsilon $][\mathrm{I}, \mathrm{J}, \mathrm{K}, \mathrm{L}] \longrightarrow \epsilon_{I J K L}$.

\footnotetext{
${ }^{13}$ The same for the other Lorentz indices $\nu_{i}$ and $\sigma_{i}$ in the WiLE output.
} 
6. The reduced vector couplings $n_{i}$ and $\bar{n}_{i}$

- Out[1]= $\mathrm{n}_{\mathrm{i}} \longrightarrow \mathrm{nJ}[\backslash[\mathrm{Tau}][\mathrm{i}]] \quad \longrightarrow \quad n_{i}$,

- Out[1]= $\mathrm{n}_{\mathrm{i} \mathrm{J}} \longrightarrow \mathrm{nJ}[\backslash[\mathrm{Tau}][\mathrm{i}], \mathrm{J}] \longrightarrow n_{i J}$,

- $\mathrm{Out}[1]=\overline{\mathrm{n}}_{\mathrm{i}} \longrightarrow \mathrm{nJb}[\backslash[\mathrm{Tau}][\mathrm{i}]] \quad \longrightarrow \quad \bar{n}_{i}$

- Out[1] $=\overline{\mathrm{n}}_{i}^{\mathrm{J}} \longrightarrow \mathrm{nJb}[\backslash[\mathrm{Tau}][\mathrm{i}], \mathrm{J}] \quad \longrightarrow \quad \bar{n}_{i}{ }^{J}$.

7. The Grassman even fermionic couplings $\eta_{i}$ and $\bar{\eta}_{i}$

- Out[1]= $\eta_{i} \rightarrow \backslash[\mathrm{Eta}][\mathrm{x}[\backslash[\mathrm{Tau}][\mathrm{i}]]] \quad \longrightarrow \quad \eta_{i}$

- $\operatorname{Out}[1]=\bar{\eta}_{i} \longrightarrow \backslash[\mathrm{Eta}] \mathrm{b}[\mathrm{x}[\backslash[\mathrm{Tau}][\mathrm{i}]]] \rightarrow \bar{\eta}_{i}$.

8. The scalar couplings $M_{i}$ and $\hat{M}_{i}$

- Out[1]= $\mathrm{M}_{\mathrm{i}} \longrightarrow \mathrm{M}[\backslash[\mathrm{Tau}][\mathrm{i}]] \quad \longrightarrow \quad \mathcal{M}_{i}$ or $M_{i}$,

- Out[1]= $\mathrm{M}_{\mathrm{iJ}}{ }^{\mathrm{K}} \longrightarrow \mathrm{M}[\backslash[\mathrm{Tau}][\mathrm{i}], \mathrm{J}, \mathrm{K}] \longrightarrow\left(\mathcal{M}_{i}\right)_{J}{ }^{K}$ or $\left(M_{i}\right)_{J}{ }^{K}$,

- Out[1]= $\hat{\mathbb{M}}_{i} \longrightarrow \operatorname{Mh}[\backslash[\mathrm{Tau}][\mathrm{i}]] \quad \longrightarrow \quad \hat{\mathcal{M}}_{i}$ or $\hat{M}_{i}$,

- $\mathrm{Out}[1]=\hat{\mathrm{M}}_{\mathrm{iJ}}{ }^{\mathrm{K}} \longrightarrow \operatorname{Mh}[\backslash[\mathrm{Tau}][\mathrm{i}], \mathrm{J}, \mathrm{K}] \longrightarrow\left(\hat{\mathcal{M}}_{i}\right)_{J}{ }^{K}$ or $\quad\left(\hat{M}_{i}\right)_{J}{ }^{K}$.

9. The propagator $\Delta$

- $\operatorname{Out}[1]=\Delta[\mathrm{x}, \mathrm{y}] \longrightarrow \backslash$ Delta $][\mathrm{x}, \mathrm{y}] \longrightarrow \Delta(x, y)$.

10. The derivative $\partial_{x}^{\mu}$

- Out[1]= $\mathrm{d}_{\mathrm{x}}{ }^{\mu}[\mathrm{f}[\mathrm{x}]] \quad \longrightarrow \mathrm{d}[\mathrm{x}, \backslash[\mathrm{Mu}], \mathrm{f}[\mathrm{x}]] \quad \longrightarrow \partial_{x}^{\mu} f(x)$.

11. The product of matrices

- Out $[1]=\mathrm{A} \cdot \mathrm{B} \cdot \mathrm{C} \longrightarrow$ Centerdot $[\mathrm{A}, \mathrm{B}, \mathrm{C}] \longrightarrow(A B C)$.

12. The trace $\operatorname{tr}$

- $\operatorname{Out}[1]=\operatorname{tr}[\mathrm{A}] \quad \longrightarrow \operatorname{tr}[\mathrm{A}] \longrightarrow \operatorname{tr}(A)$

\section{References}

[1] A. M. Polyakov, "Gauge Fields as Rings of Glue", Nucl. Phys. B164, 171 (1980).

[2] G. P. Korchemsky and A. V. Radyushkin, "Infrared factorization, Wilson lines and the heavy quark limit”, Phys. Lett. B279, 359 (1992), hep-ph/9203222.

[3] G. P. Korchemsky and G. Marchesini, "Resummation of large infrared corrections using Wilson loops", Phys. Lett. B313, 433 (1993).

[4] J. M. Maldacena, "The Large N limit of superconformal field theories and supergravity", Adv.Theor.Math.Phys. 2, 231 (1998), hep-th/9711200.

[5] E. Witten, "Anti-de Sitter space and holography", Adv. Theor. Math. Phys. 2, 253 (1998), hep-th/9802150. 
[6] S. S. Gubser, I. R. Klebanov and A. M. Polyakov, "Gauge theory correlators from non-critical string theory", Phys. Lett. B428, 105 (1998), hep-th/9802109.

[7] J. M. Maldacena, “Wilson loops in large N field theories", Phys. Rev. Lett. 80, 4859 (1998), hep-th/9803002.

[8] S.-J. Rey and J.-T. Yee, "Macroscopic strings as heavy quarks in large $N$ gauge theory and anti-de Sitter supergravity", Eur.Phys.J. C22, 379 (2001), hep-th/9803001.

[9] J. K. Erickson, G. W. Semenoff and K. Zarembo, "Wilson loops in $N=4$ supersymmetric Yang-Mills theory", Nucl. Phys. B582, 155 (2000), hep-th/0003055.

[10] N. Drukker and D. J. Gross, "An Exact prediction of N=4 SUSYM theory for string theory", J. Math. Phys. 42, 2896 (2001), hep-th/0010274.

[11] L. J. Mason and D. Skinner, "The Complete Planar S-matrix of N=4 SYM as a Wilson Loop in Twistor Space", JHEP 1012, 018 (2010), arXiv:1009.2225.

[12] J. M. Drummond, J. Henn, G. P. Korchemsky and E. Sokatchev, "On planar gluon amplitudes/Wilson loops duality", Nucl. Phys. B795, 52 (2008), arXiv:0709.2368.

[13] S. Caron-Huot, "Notes on the scattering amplitude / Wilson loop duality", JHEP 1107, 058 (2011), arXiv: 1010.1167.

[14] L. F. Alday and R. Roiban, "Scattering Amplitudes, Wilson Loops and the String/Gauge Theory Correspondence", Phys. Rept. 468, 153 (2008), arXiv:0807.1889.

[15] R. M. Schabinger, "One-loop $N=4$ super Yang-Mills scattering amplitudes in d dimensions, relation to open strings and polygonal Wilson loops", J. Phys. A44, 454007 (2011), arXiv:1104.3873.

[16] T. Adamo, M. Bullimore, L. Mason and D. Skinner, "Scattering Amplitudes and Wilson Loops in Twistor Space", J. Phys. A44, 454008 (2011), arXiv:1104.2890.

[17] S. Giombi and V. Pestun, "Correlators of local operators and 1/8 BPS Wilson loops on $S^{* * 2}$ from 2d YM and matrix models", JHEP 1010, 033 (2010), arXiv:0906.1572.

[18] S. Giombi and V. Pestun, "Correlators of Wilson Loops and Local Operators from Multi-Matrix Models and Strings in AdS", JHEP 1301, 101 (2013), arXiv:1207.7083.

[19] M. Bonini, L. Griguolo and M. Preti, "Correlators of chiral primaries and 1/8 BPS Wilson loops from perturbation theory”, JHEP 1409, 083 (2014), arXiv:1405.2895.

[20] M. Preti, "Studies on Wilson loops, correlators and localization in supersymmetric quantum field theories".

[21] N. Drukker, "Integrable Wilson loops", JHEP 1310, 135 (2013), arXiv:1203.1617.

[22] D. Correa, J. Maldacena and A. Sever, "The quark anti-quark potential and the cusp anomalous dimension from a TBA equation", JHEP 1208, 134 (2012), arXiv:1203.1913.

[23] M. Bonini, L. Griguolo, M. Preti and D. Seminara, "Bremsstrahlung function, leading Lüscher correction at weak coupling and localization", JHEP 1602, 172 (2016), arXiv:1511.05016.

[24] M. Cooke, A. Dekel and N. Drukker, "The Wilson loop CFT: Insertion dimensions and structure constants from wavy lines", arXiv:1703.03812.

[25] S. Giombi, R. Roiban and A. A. Tseytlin, "Half-BPS Wilson loop and $A d S_{2} / C F T_{1}$ ", arXiv:1706.00756.

[26] O. Aharony, O. Bergman, D. L. Jafferis and J. Maldacena, "NN=6 superconformal Chern-Simons-matter theories, M2-branes and their gravity duals", JHEP 0810, 091 (2008), arXiv:0806.1218. 
[27] O. Aharony, O. Bergman and D. L. Jafferis, "Fractional M2-branes", JHEP 0811, 043 (2008), arXiv: 0807.4924.

[28] N. Drukker, J. Plefka and D. Young, "Wilson loops in 3-dimensional N=6 supersymmetric Chern-Simons Theory and their string theory duals", JHEP 0811, 019 (2008), arXiv:0809.2787.

[29] B. Chen and J.-B. Wu, "Supersymmetric Wilson Loops in N=6 Super Chern-Simons-matter theory", Nucl. Phys. B825, 38 (2010), arXiv:0809.2863.

[30] S.-J. Rey, T. Suyama and S. Yamaguchi, "Wilson Loops in Superconformal Chern-Simons Theory and Fundamental Strings in Anti-de Sitter Supergravity Dual”, JHEP 0903, 127 (2009), arXiv:0809.3786.

[31] N. Drukker and D. Trancanelli, "A Supermatrix model for N=6 super Chern-Simons-matter theory", JHEP 1002, 058 (2010), arXiv:0912.3006.

[32] K.-M. Lee and S. Lee, "1/2-BPS Wilson Loops and Vortices in ABJM Model", JHEP 1009, 004 (2010), arXiv:1006.5589.

[33] D. Berenstein and D. Trancanelli, "Three-dimensional $\mathcal{N}=6$ SCFT's and their membrane dynamics", Phys. Rev. D78, 106009 (2008), arXiv:0808.2503.

[34] M. S. Bianchi, G. Giribet, M. Leoni and S. Penati, "The 1/2 BPS Wilson loop in ABJM theory at two loops", Phys.Rev. D88, 026009 (2013), arXiv:1303.6939.

[35] L. Griguolo, G. Martelloni, M. Poggi and D. Seminara, "Perturbative evaluation of circular 1/2 BPS Wilson loops in $\mathcal{N}=6$ Super Chern-Simons theories", JHEP 1309, 157 (2013), arXiv: 1307.0787.

[36] M. Bianchi, G. Giribet, M. Leoni and S. Penati, "The 1/2 BPS Wilson loop in ABJ(M) at two loops: The details", JHEP 1310, 085 (2013), arXiv:1307.0786.

[37] L. Griguolo, D. Marmiroli, G. Martelloni and D. Seminara, "The generalized cusp in $A B J(M) N=$ 6 Super Chern-Simons theories", JHEP 1305, 113 (2013), arXiv:1208.5766.

[38] L. Bianchi, M. S. Bianchi, A. Bres, V. Forini and E. Vescovi, "Two-loop cusp anomaly in ABJM at strong coupling", JHEP 1410, 013 (2014), arXiv:1407.4788.

[39] M. Bonini, L. Griguolo, M. Preti and D. Seminara, "Surprises from the resummation of ladders in the ABJ(M) cusp anomalous dimension", JHEP 1605, 180 (2016), arXiv:1603.00541.

[40] M. S. Bianchi, L. Griguolo, M. Leoni, S. Penati and D. Seminara, "BPS Wilson loops and Bremsstrahlung function in ABJ(M): a two loop analysis", JHEP 1406, 123 (2014), arXiv: 1402.4128.

[41] M. S. Bianchi, L. Griguolo, A. Mauri, S. Penati, M. Preti and D. Seminara, "Towards the exact Bremsstrahlung function of ABJM theory", arXiv:1705.10780.

[42] L. Bianchi, L. Griguolo, M. Preti and D. Seminara, "Wilson lines as superconformal defects in ABJM theory: a formula for the emitted radiation", arXiv:1706.06590.

[43] D. Gaiotto and X. Yin, "Notes on superconformal Chern-Simons-Matter theories", JHEP 0708, 056 (2007), arXiv:0704.3740.

[44] V. Cardinali, L. Griguolo, G. Martelloni and D. Seminara, "New supersymmetric Wilson loops in ABJ(M) theories", Phys. Lett. B718, 615 (2012), arXiv:1209.4032.

[45] K. Zarembo, "Supersymmetric Wilson loops", Nucl. Phys. B643, 157 (2002), hep-th/0205160.

[46] N. Drukker, S. Giombi, R. Ricci and D. Trancanelli, "More supersymmetric Wilson loops", Phys. Rev. D76, 107703 (2007), arXiv:0704.2237. 
[47] N. Drukker, S. Giombi, R. Ricci and D. Trancanelli, "Supersymmetric Wilson loops on $S^{* * 3 ", ~}$ JHEP 0805, 017 (2008), arXiv: 0711.3226.

[48] E. Witten, "Quantum Field Theory and the Jones Polynomial", Commun. Math. Phys. 121, 351 (1989).

[49] E. Guadagnini, M. Martellini and M. Mintchev, "Wilson Lines in Chern-Simons Theory and Link Invariants", Nucl.Phys. B330, 575 (1990).

[50] M. Alvarez and J. M. F. Labastida, "Analysis of observables in Chern-Simons perturbation theory", Nucl. Phys. B395, 198 (1993), hep-th/9110069.

[51] N. Drukker, M. Marino and P. Putrov, "From weak to strong coupling in ABJM theory", Commun. Math. Phys. 306, 511 (2011), arXiv:1007.3837.

[52] D. Correa, J. Henn, J. Maldacena and A. Sever, "The cusp anomalous dimension at three loops and beyond", JHEP 1205, 098 (2012), arXiv:1203.1019.

[53] T. Huber and D. Maitre, "HypExp 2, Expanding Hypergeometric Functions about Half-Integer Parameters", Comput. Phys. Commun. 178, 755 (2008), arXiv:0708.2443.

[54] M. A. Bandres, A. E. Lipstein and J. H. Schwarz, "Studies of the ABJM Theory in a Formulation with Manifest SU(4) R-Symmetry", JHEP 0809, 027 (2008), arXiv:0807.0880. 